\title{
Design of high gradient, high repetition rate damped $C$-band rf structures
}

\author{
David Alesini, Marco Bellaveglia, Simone Bini, Alessandro Gallo, \\ Valerio Lollo, Luigi Pellegrino, and Luca Piersanti \\ LNF-INFN, Via E. Fermi 40, 00044 Frascati, Rome, Italy \\ Fabio Cardelli, Mauro Migliorati, Andrea Mostacci, Luigi Palumbo, and Simone Tocci \\ University of Rome "La Sapienza"-SBAI Department, Via A. Scarpa 14, 00161 Rome, Italy \\ Luca Ficcadenti and Valerio Pettinacci \\ INFN Roma, Piazzale A. Moro 2, 00185 Rome, Italy \\ (Received 22 June 2016; published 8 March 2017)
}

\begin{abstract}
The gamma beam system of the European Extreme Light Infrastructure-Nuclear Physics project foresees the use of a multibunch train colliding with a high intensity recirculated laser pulse. The linac energy booster is composed of 12 traveling wave $C$-band structures, $1.8 \mathrm{~m}$ long with a field phase advance per cell of $2 \pi / 3$ and a repetition rate of $100 \mathrm{~Hz}$. Because of the multibunch operation, the structures have been designed with a dipole higher order mode (HOM) damping system to avoid beam breakup (BBU). They are quasiconstant gradient structures with symmetric input couplers and a very effective damping of the HOMs in each cell based on silicon carbide ( $\mathrm{SiC}$ ) rf absorbers coupled to each cell through waveguides. An optimization of the electromagnetic and mechanical design has been done to simplify the fabrication and to reduce the cost of the structures. In the paper, after a review of the beam dynamics issues related to the BBU effects, we discuss the electromagnetic and thermomechanic design criteria of the structures. We also illustrate the criteria to compensate the beam loading and the $\mathrm{rf}$ measurements that show the effectiveness of the HOM damping.
\end{abstract}

DOI: 10.1103/PhysRevAccelBeams.20.032004

\section{INTRODUCTION}

An advanced source of gamma-ray photons will be built in Magurele (Bucharest, Romania) in the context of the Extreme Light Infrastructure-Nuclear Physics (ELI-NP) Research Infrastructure [1]. The photons will be generated by Compton backscattering in the collision between a high quality electron beam and a high power laser. The machine is expected to achieve an energy of the gamma photons tunable between 0.2 and $19.5 \mathrm{MeV}$ with a narrow bandwidth $(<0.5 \%)$ and a high spectral density $\left(>10^{4}\right.$ photons $\left./ \mathrm{sec} / \mathrm{eV}\right)$. The machine is based on a $\mathrm{rf}$ linac operated at $C$-band $(5.712 \mathrm{GHz})$ with an S-band photoinjector similar to the SPARC_LAB one at LNFINFN (Frascati, Rome) [2], delivering a high phase space density electron beam in the $80-740 \mathrm{MeV}$ energy range. The repetition rate of the machine is $100 \mathrm{~Hz}$ and, within the rf pulse, up to 32 electron bunches will be accelerated, each one carrying $250 \mathrm{pC}$ of charge, separated by $16 \mathrm{~ns}$. The linac booster is composed of 12 TW $C$-band disk

Published by the American Physical Society under the terms of the Creative Commons Attribution 4.0 International license. Further distribution of this work must maintain attribution to the author(s) and the published article's title, journal citation, and DOI. loaded accelerating structures; each structure, $1.8 \mathrm{~m}$ long, is a quasiconstant gradient structure with $2 \pi / 3$ phase advance per cell and will operate at an average accelerating gradient of $33 \mathrm{MV} / \mathrm{m}$ with $40 \mathrm{MW}$ of input power. The main electron beam and $C$-band cavity parameters of the ELI-NP gamma beam system (GBS) are given in Table I. Because of the multibunch operation, the structures have been designed with a damping system to avoid beam breakup (BBU) instabilities. Moreover, since the average dissipated power in the structure is $2.3 \mathrm{~kW}$, the cooling system has been integrated into the structure itself and it has been dimensioned in order to avoid structure detuning under $\mathrm{rf}$ operation. With respect to already fabricated $C$-band structures like the Spring 8 ones [3-5] the ELI-NP structures have a higher shunt impedance and can operate at a higher repetition rate and in multibunch, i.e. at an higher average dissipated power. In the first section of the paper we report the main results related to the multibunch issues and BBU analysis. The electromagnetic design of the structures is illustrated in the second section while the vacuum and thermal analysis results are given in the third section. The fourth section is dedicated to the illustration of the beam loading analysis while, in the last section, we report the results of the rf measurements on a prototype that show the effectiveness of the $\mathrm{SiC}$ absorbers in the HOM damping. 
TABLE I. Main electron beam and $C$-band cavity parameters of the ELI-NP GBS.

\begin{tabular}{lc}
\hline \hline Electron beam energy [MeV] & $80-740$ \\
Normalized beam emittance [mm mrad] & $<0.5$ \\
Number of colliding bunches & 32 \\
Bunch spacing [ns] & 16 \\
Bunch charge [pC] & 250 \\
Repetition rate [Hz] & 100 \\
$C$-band cavity frequency [GHz] & 5.712 \\
Average accelerating field [MV/m] & 33 \\
Structure length [m] & 1.8 \\
Working mode & $2 \pi / 3$ \\
rf input power [MW] & 40 \\
\hline \hline
\end{tabular}

\section{BBU ISSUES IN THE $\boldsymbol{C}$-BAND STRUCTURES}

Long range wakefields, in multibunch operation, can strongly affect the accelerator beam dynamics. The longitudinal ones, related to the excitation of the fundamental accelerating mode and referred to as beam loading effects, can give a modulation of the beam energy along the bunch trains. The strategy to compensate the beam loading will be discussed in Sec. V.

Of particular concern in the ELI-NP linac is the multibunch BBU driven by the transverse wakefields [6]. Because of the tight requirements on the narrow bandwidth of the gamma ray photon beam, which in turn asks for very low transverse emittances, we have carefully evaluated the emittance degradation due to BBU. As a bunch in a beam pulse is displaced from the axis, transverse deflecting dipole modes are excited. The trailing bunches are then deflected by the wakefield forces whether they are on axis or not. The angular deflections transform into displacements through the transfer matrices of the focusing system and these displaced bunches will themselves create wakefields in the downstream cavities of the linac. The subsequent bunches will be further deflected leading to a beam blowup [6-9].

Off-axis beam trajectories, the source of the BBU effect, arise due to a variety of errors like offset at injection, misalignment of focusing magnets and misalignment of accelerating sections. The beam can excite several dipole modes at different frequencies but, in general, the contribution of the dipole modes at the lower frequency dominates $[10,11]$. The beam dynamics simulations and the modeling of such phenomena can be done following different models and, in particular, in the ELI-NP case we have used two independent approaches: (i) an analytical approach based on the Mosnier theory [6] in which the bunches are considered to be rigid macroparticles, like delta functions, injected with the same initial offset $x_{0}$ (in this approach only the fundamental dipole mode can be considered and the $\beta$-function is considered constant along the linac); (ii) a tracking code approach in which the bunches are still considered to be rigid macroparticles which, differently from the analytical model, allows a more complete analysis taking into account the contribution of several resonant modes, different initial offsets of the bunches, different charge per bunch and not uniform $\beta$-function along the linac. As shown in $[1,12]$ the two approaches give similar results in the simple case of single resonant mode, equal initial offset for all bunches and constant $\beta$-function.

An example of the tracking code result is given in Fig. 1 where the normalized Courant-Snyder invariants $\left(\mathrm{I}_{n}\right)$ of each bunch at the exit of the linac are plotted for an initial displacement at the entrance of the linac for all bunches equal to $200 \mu \mathrm{m}$. For each bunch we have defined $\mathrm{I}_{n}$ as follows:

$$
I_{n}=\frac{\frac{1}{\beta}\left(x_{n}-x_{1}\right)^{2}+\beta\left(x_{n}^{\prime}-x_{1}^{\prime}\right)^{2}}{\gamma},
$$

where $\mathrm{x}_{n}, \mathrm{x}_{n}^{\prime}$ are the output position and angle of the $n$th bunch and $\gamma$ is the relativistic factor of the beam. To evaluate the effect of the BBU, the simulations have been done assuming $C$-band structures similar to those fabricated for the SPARC_LAB energy upgrade [13]. The result is referred to the case of a single dipole mode with the parameters given in Table II. The transverse shunt impedance of the dipole mode $\mathrm{R}_{T}$ has been defined as:

$$
R_{T}=\frac{\left|\int_{0}^{L_{c}} E_{T} e^{j \omega_{\mathrm{res}} z / c} d z\right|^{2}}{2 P_{\mathrm{diss}}}[\Omega],
$$

where $\mathrm{E}_{T}$ is the equivalent transverse deflecting field (given by the Lorentz transverse force $\mathrm{F}_{T}$ divided by the charge), $\mathrm{L}_{c}$ is the single cell length and $\mathrm{P}_{\text {diss }}$ is the dissipated power in the cell. In the calculations we have considered an injection

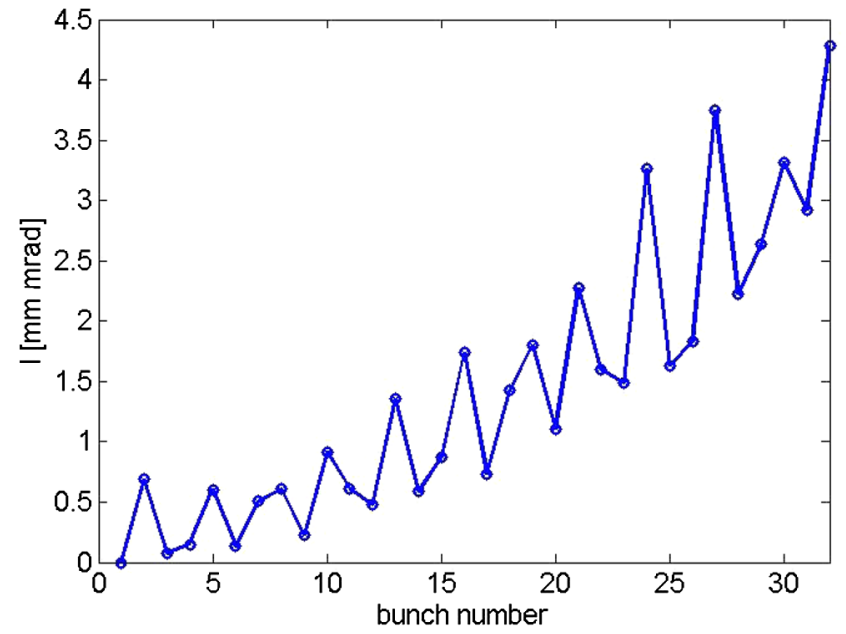

FIG. 1. Normalized Courant Snyder invariants at the exit of the linac for an initial displacement of all bunches of $200 \mu \mathrm{m}$ (in the calculation we have considered an average accelerating field of $28 \mathrm{MV} / \mathrm{m}$ that includes the drift spaces, diagnostics etc.). 
TABLE II. First dipole mode and $C$-band linac parameters used in the evaluation of the BBU effects (the transverse shunt impedance $\mathrm{R}_{T}$ is per cell, $\mathrm{Q}$ is the quality factor of the dipole mode).

\begin{tabular}{lc}
\hline \hline $\mathrm{R}_{T} / \mathrm{Q}$ & $26 \Omega$ \\
$\mathrm{Q}$ & 11000 \\
$\mathrm{f}_{\text {res }}$ & $8.398 \mathrm{GHz}$ \\
$\mathrm{R} / \mathrm{Q}\left(=\omega_{\text {res }} / \mathrm{c} * \mathrm{R}_{T} / \mathrm{Q} / \mathrm{L}_{c}\right)$ & $0.26 \mathrm{M} \Omega / \mathrm{m}^{2}$ \\
$\mathrm{E}_{\text {inj }}$ & $80 \mathrm{MeV}$ \\
$\mathrm{E}_{\text {acc }}$ & $33 \mathrm{MV} / \mathrm{m}$ \\
$\beta$ & $5 \mathrm{~m}$ \\
\hline \hline
\end{tabular}

energy at the entrance of the linac $\left(\mathrm{E}_{\text {inj }}\right)$ equal to $80 \mathrm{MeV}$ (that corresponds to the energy at the exit of the ELI-NP S-band injector), an average $\beta$-function of $5 \mathrm{~m}$ and an average accelerating gradient of $28 \mathrm{MV} / \mathrm{m}$.

The Courant-Snyder invariants plotted in Fig. 1 can be directly compared with the nominal normalized emittance of bunches $(0.4 \mathrm{~mm} \mathrm{mrad})$. The result clearly shows that the BBU increases the final projected emittance of the train and cannot be tolerated. Moreover this result strongly depends on the resonant frequency of the dipole mode and even worse results can be obtained by slightly changing this resonant frequency.

As discussed in the next section, several solutions could be adopted to mitigate the BBU, like the damping and/or detuning [14]. For the ELI-NP structures we have proposed to adopt a more drastic solution based on a strong damping of such dipole mode similar to the X-band CLIC structures [15]. To estimate the maximum quality factor necessary to completely suppress the BBU instability, we have calculated the maximum $\mathrm{I}_{n}$ as a function of the first dipole mode

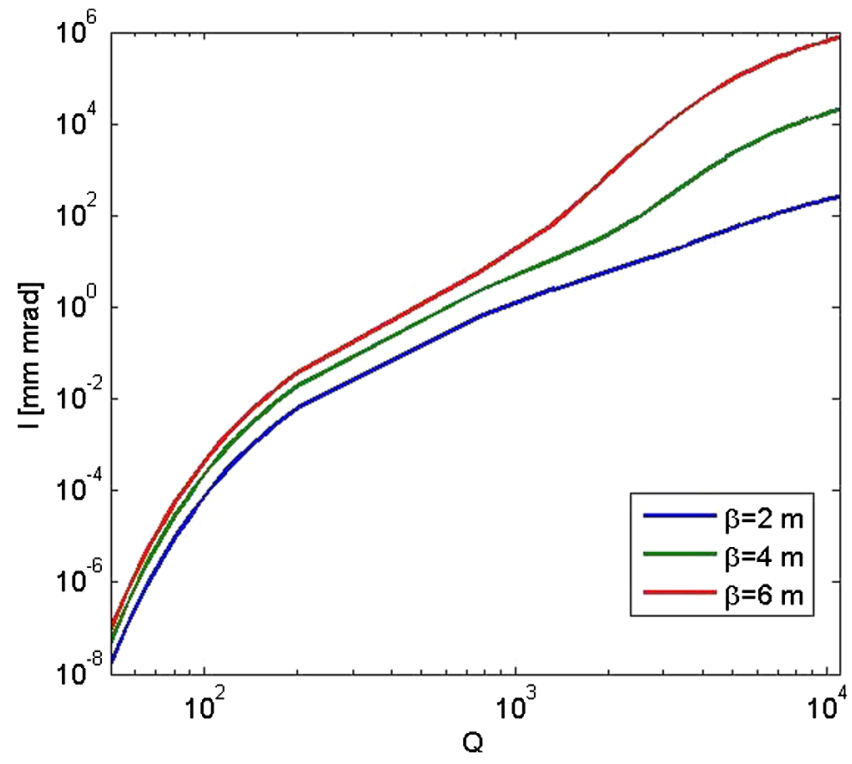

FIG. 2. Maximum value of the normalized Courant Snyder invariants at the end of the $C$-band linac as a function of the first dipole mode quality factor and for different $\beta$-functions (initial displacement of all bunches: $200 \mu \mathrm{m}$, perfect transverse wake buildup).

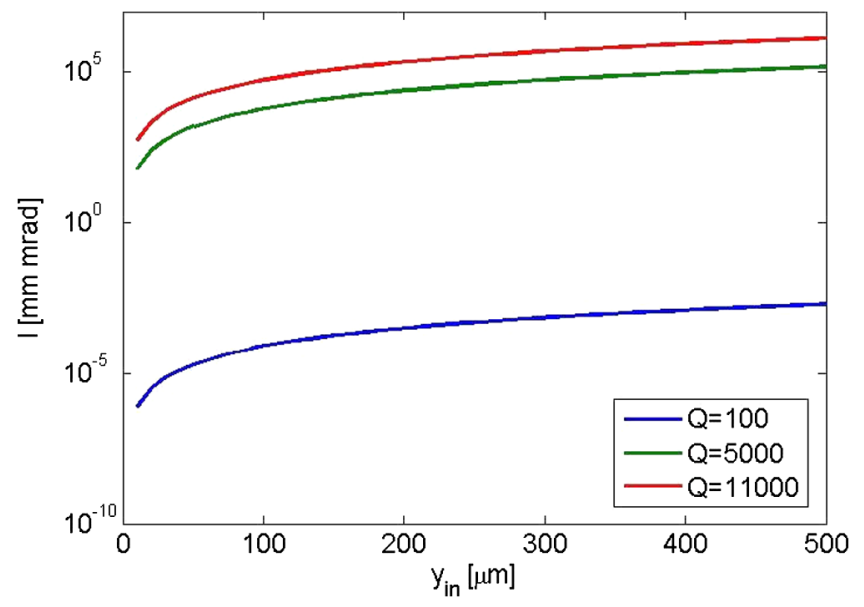

FIG. 3. Maximum value of the normalized Courant Snyder invariants at the end of the $C$-band linac as a function of the injection errors and for different Q-factors of the first dipole modes (perfect transverse wake buildup).

quality factor, for different injection errors and for different average $\beta$-functions. Moreover, in the calculation we have considered a perfect buildup mechanism of the transverse wakes to have an estimation of the BBU effects in the worst case. The results are given in Figs. 2 and 3 and clearly show that, with a quality factor below 100, the BBU is completely suppressed. Similar considerations can be done for the other dipole HOM at higher frequencies. The estimation of a quality factor below 100 is also pessimistic because we have considered a perfect buildup mechanism of the wake. This means that, with this HOM damping system, we have the possibility to increase the average beam current, and this allows one to use such a structure also in future machine upgrades at higher current.

In conclusion, the design of the $C$-band sections has been addressed to reach an overall quality factor of the dipole modes below 100 to avoid BBU instabilities. This value has been considered as a design parameter.

\section{ELECTROMAGNETIC STRUCTURE DESIGN}

The overall design of the structure has been addressed to simultaneously obtain: (a) an average accelerating gradient of $33 \mathrm{MV} / \mathrm{m}$ with an available power from the klystron of $40 \mathrm{MW}$; (b) a quality factor of the dipole modes below 100 to avoid BBU instabilities as pointed out in the previous section; (c) the lowest peak surface electric field on the irises; (d) the largest iris aperture compatible with the previous points to increase the pumping speed of the structure, to reduce the dipole wakefield intensity and the filling time of the structure itself. The reduction of this last parameter allows reaching higher accelerating gradient since shorter rf pulse length reduces the breakdown rate.

Based on these considerations the electromagnetic design of the structure has been done in three steps: (a) HOM damping system; (b) irises cell; (c) input/output couplers. 


\section{A. HOM damping system design approach}

As already mentioned there are basically two methods to damp the dipole modes in the accelerating cells: with waveguides opened in each cell and coupled to dipole modes (like in the CLIC X-band structures [15]), or with the choke mode technique proposed and adopted for the Spring 8 structures [3-5]. In the first case, the beam excited dipole fields propagate into the waveguides and are dissipated into $\mathrm{rf}$ absorbers, while the fundamental mode is rejected because it is below the cutoff frequency of the waveguides themselves. In the second approach the cells are opened and all dipole fields can propagate into if absorbers while the fundamental accelerating mode is rejected by a notch filter inserted between the cells and the rf absorbers. Both approaches can give a strong damping of the dipole modes and have advantages and disadvantages. In the first case (because of the presence of the waveguides) the cells have to be machined with a 3D milling machine, while in the second case the cells can be machined by a lathe. On the other hand, in this second case, the presence of the choke reduces the shunt impedance of the cells with respect to the first case. Moreover, in the first case, the cells can be easily tuned and the pipes for the cooling system can be located near the internal surface of the cells allowing a better temperature control and stabilization that is crucial in the high repetition rate and long rf pulses operation (like in the ELI-NP case).

For these reasons we have adopted a dipole damping system based on waveguides, but the geometry of the dampers and the overall mechanical design of the cells have been strongly simplified with respect to the CLIC case to reduce the cost, to simplify the fabrication, to reduce the risk of failure in the realization and brazing processes and the delivery time [16], as illustrated in the following paragraphs.

\section{B. HOM damping system implementation}

Starting with a simple pill box cavity (with irises), operating on the $2 \pi / 3 \mathrm{TW}$ accelerating mode, the cell dimensions have been initially calculated to obtain (in the case of all equal cells and a $1.8 \mathrm{~m}$ long structure) an average

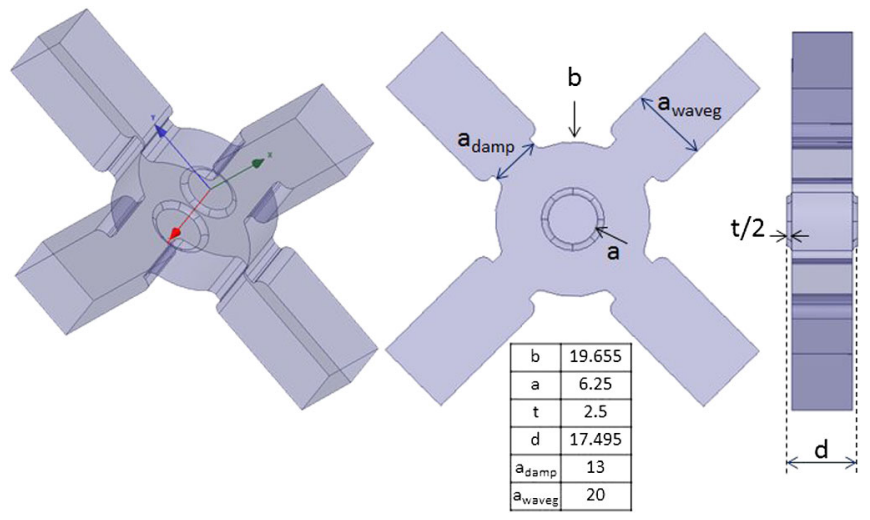

FIG. 4. HFSS single cell geometry with main cell dimensional parameters (table dimensions in $\mathrm{mm}$ ). accelerating field of $33 \mathrm{MV} / \mathrm{m}$ with an available power $\left(\mathrm{P}_{\mathrm{IN}}\right)$ from the klystron equal to $40 \mathrm{MW}$.

Four waveguides (shown in Fig. 4) have been opened in each cell of the structure allowing the excited HOMs to propagate and dissipate into silicon-carbide $(\mathrm{SiC}) \mathrm{rf}$ absorbers. The waveguide widths ( $a_{\text {waveg }}$ ) have been chosen to allow the propagation of the first dipole mode while the waveguide apertures $\left(\mathrm{a}_{\text {damp }}\right)$ have been optimized to obtain a quality factor of the dipole modes below 100 or, equivalently, a negligible transverse wakefield contribution after $16 \mathrm{~ns}$. To this purpose, frequency domain simulations, using the commercial code HFSS [17], and time domain simulations using the commercial codes GDFIDL [18] and CST MICROWAVE STUDIO [19] have been performed. The HFSS single cell geometry is given in Fig. 4 with its main geometrical parameters. In HFSS the waveguide apertures have been optimized using the driven mode solver and analyzing the transmission coefficient between the damping waveguides as a function of $\mathrm{a}_{\mathrm{damp}}$. As an example the transmission coefficient between two waveguides of a fivecell structure is given in Fig. 5 for two different waveguide apertures and in the frequency interval corresponding to the first dipole band. In this case with an $\mathrm{a}_{\mathrm{damp}}=7 \mathrm{~mm}$ (blue curve) the quality factors of the modes are about 3000 while, in the case of $\mathrm{a}_{\mathrm{damp}}=13 \mathrm{~mm}$ (red curve), they are so low that the modes cannot be distinguished because their bandwidths are larger than 100-150 MHz which means Q < 80-50.

Similarly using the mentioned time domain simulation codes the transverse wakefield has been calculated considering a 20-cell structure and different waveguide apertures. In this case the waveguides have been considered perfectly matched. The CST simulated structure is given, as an example, in Fig. 6(a). After this design phase the waveguide apertures have been fixed to $13 \mathrm{~mm}$. The simulated CST transverse wake potential is given in Fig. 7(a) (blue curve) and clearly shows

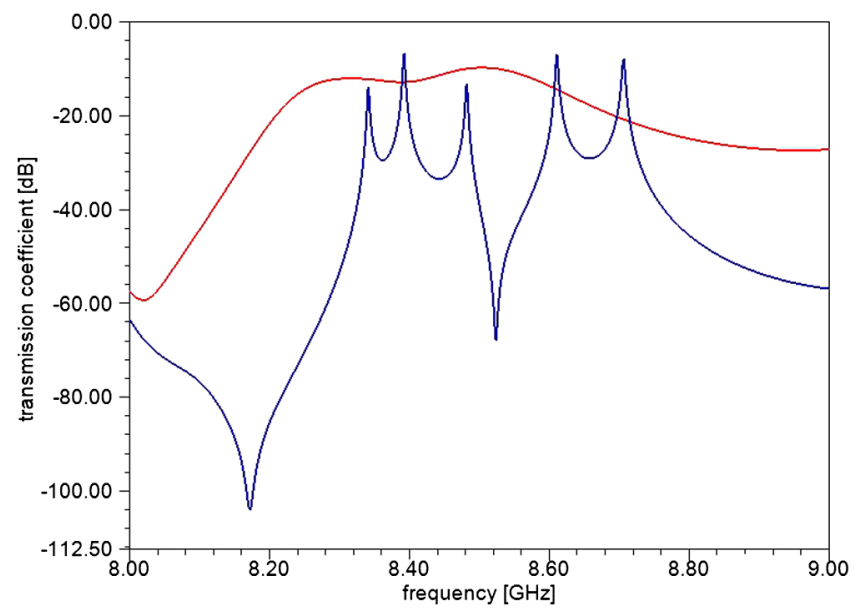

FIG. 5. HFSS simulated transmission coefficient between two waveguides of a five-cell structure for two different waveguide apertures $a_{\text {damp }}$ in the frequency interval corresponding to the first dipole band (blue curve: $\mathrm{a}_{\text {damp }}=7 \mathrm{~mm}$, red curve: $\mathrm{a}_{\mathrm{damp}}=13 \mathrm{~mm}$ ). 
(b)

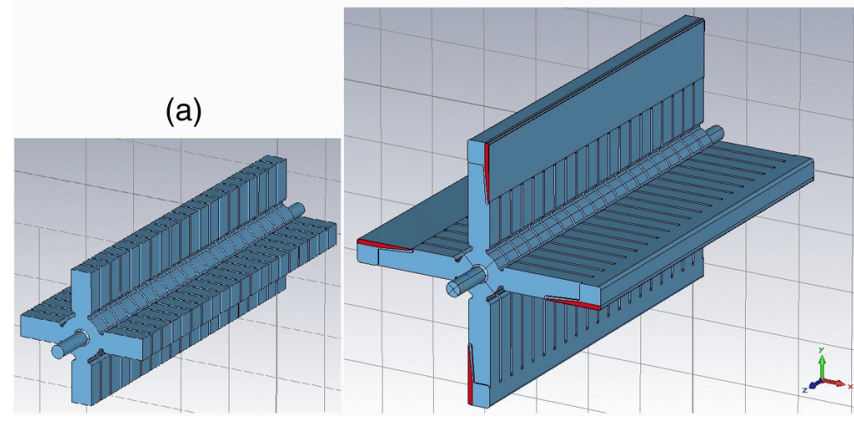

FIG. 6. CST 20-cell simulated structures with perfect matched waveguides (a) and with $\mathrm{SiC}$ absorbers (b). an attenuation of the transverse wakefield more than 2 orders of magnitude after $16 \mathrm{~ns}$ (i.e. $4.8 \mathrm{~m}$ ). The wakefield has been obtained with a beam sigma of $3 \mathrm{~mm}$, an off axis of the beam of $2 \mathrm{~mm}$ and all perfect matched ports. The corresponding transverse impedance is given in Fig. 7(b) and the quality factor of the first dipole modes are below 100, as can be calculated from the decay time of the wakefield or by the bandwidth of the corresponding impedance.

\section{SiC absorbers design}

The SiC rf absorbers have been then designed. The geometry of the $\mathrm{SiC}$ absorbers has been strongly simplified as shown in Fig. 8 in order to simplify the fabrication process,

(a)

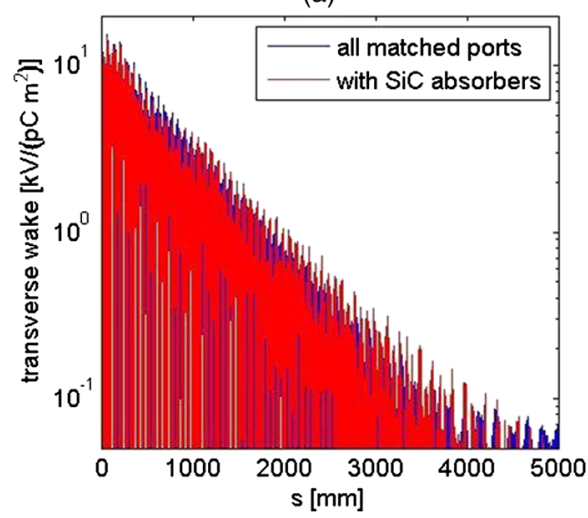

(b)

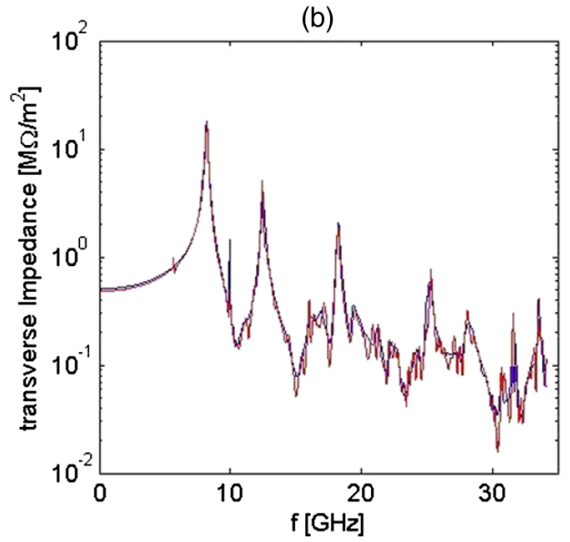

FIG. 7. (a) Transverse wake potential per unit length calculated by CST: beam sigma $3 \mathrm{~mm}$, beam off axis $2 \mathrm{~mm}$; (b) corresponding transverse impedance.

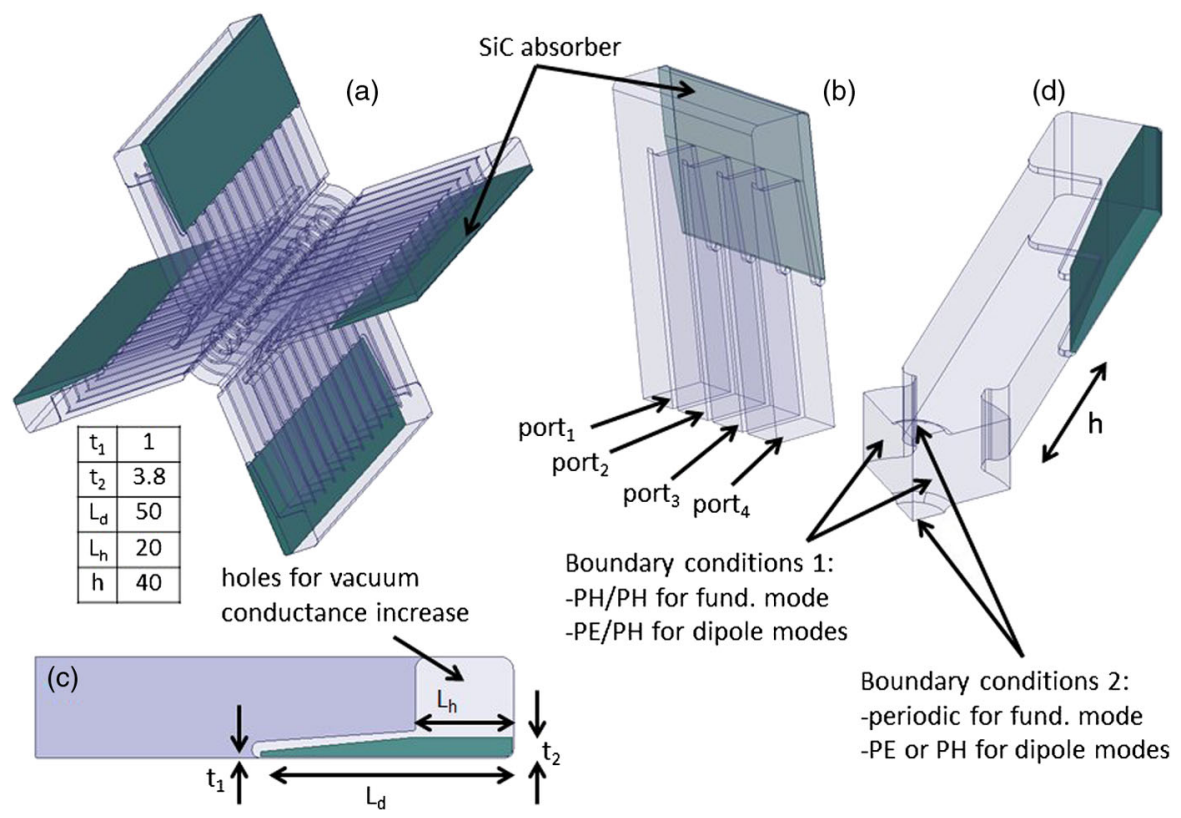

FIG. 8. HFSS simulated structures: (a) module of 12 cells with SiC absorbers; (b) absorbers; (c) main dimensions of the absorber; (d) single cell with absorber (PE means perfect electric boundary condition, PH perfect magnetic boundary condition). Table dimensions are in $\mathrm{mm}$. 
thus reducing the overall production cost and the risk of failure. The overall structure has been conceived in modules, each of 12 cells [Fig. 8(a)], with four $\mathrm{SiC}$ long tiles absorbers. Each 12-cell stack is brazed and all stacks are finally assembled and brazed with the input and output couplers. Details of the realization procedure will be discussed in an outcoming dedicated paper. From the electromagnetic point of view the $\mathrm{SiC}$ tiles have a very simple geometry that guarantees, at the same time, the rf performances. The details of the shape of the SiC absorbers are given in Fig. 8(c). The optimization of the tile profiles has been done using HFSS. We have chosen the Ekasic-P [20-22] material for its electrical properties. The HFSS simulated structure is given in Fig. 8(b). The SiC main dimensions [Fig. 8(c)] have been optimized minimizing the reflection coefficient at the waveguide input ports of the module shown in Fig. 8(b), maintaining, at the same time, a reasonable transverse dimension of the overall damper system. Moreover, large holes (shown in the figure) have been foreseen to maintain a good vacuum conductance between cells. In the simulations we have assumed the electrical properties of the Ekasic-P reported in [20]. The Ekasic-P has a quite uniform $\varepsilon_{r}$ as a function of frequency up to $20 \mathrm{GHz}$, and we have assumed a mean value of the complex dielectric constant $\left(\varepsilon_{r}\right)$ equal to $\varepsilon_{r}=2+\mathrm{j} 10$. As an example the reflection coefficients at the four input ports shown in Fig. 8(b), corresponding to the bandwidth of the first dipole modes, are given in Fig. 9. The plot clearly shows the very low reflection coefficient that allows a complete absorption of the power released by the beam to the HOM. The effectiveness of the damping has also been verified calculating the quality factor of the dipole modes of the single cell shown in Fig. 8(d). The results are summarized in Fig. 10 where the quality factors of the modes are reported as a function of the mode frequencies up to $15 \mathrm{GHz}$. When the single cell is simulated by HFSS (using the eigenmode solver), many modes appear because the code calculates both the modes confined in the

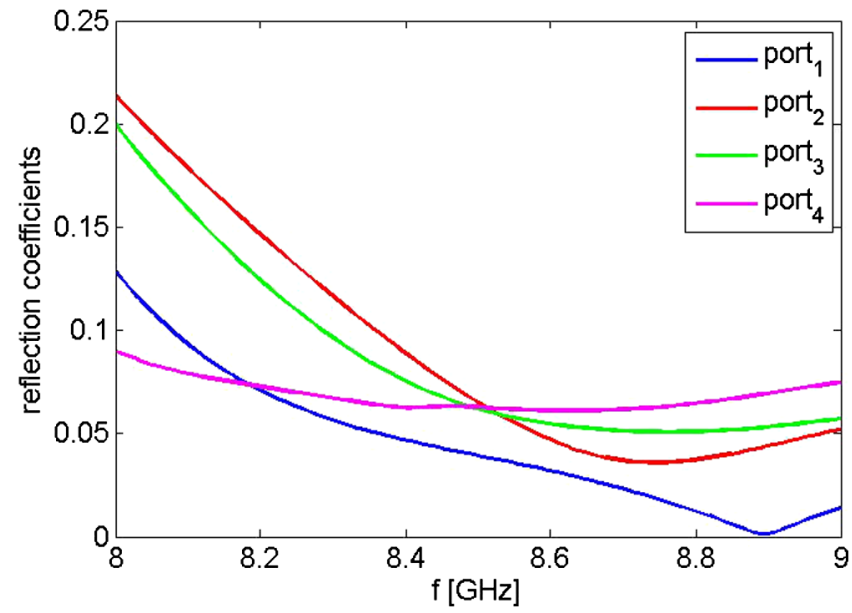

FIG. 9. Reflection coefficients at the four input ports of Fig. 8(b), corresponding to the bandwidth of the first dipole modes (HFSS simulations, port nomenclature is referred to Fig. 8).

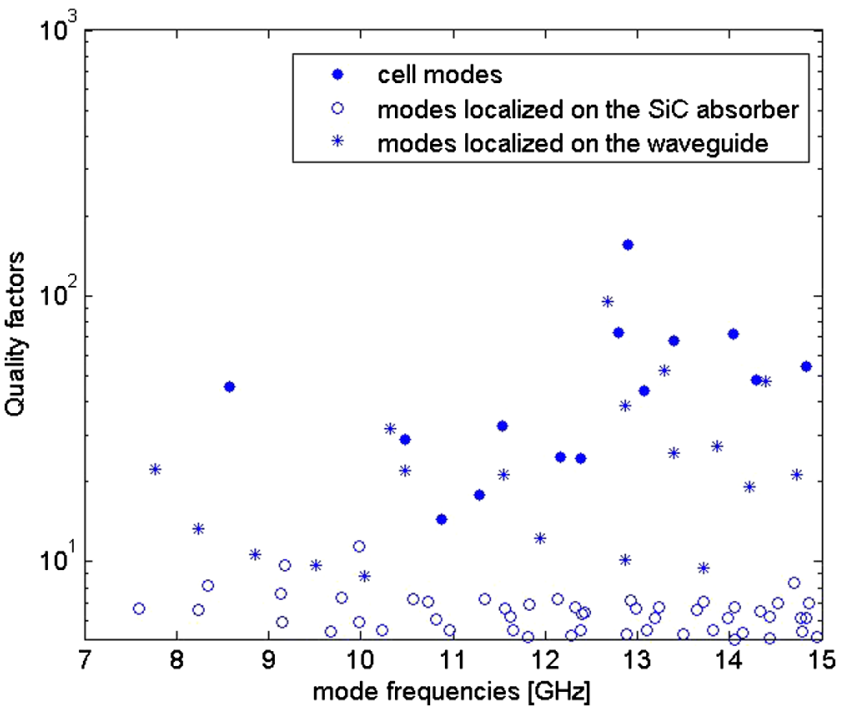

FIG. 10. Quality factors of single cell dipole modes as a function of frequency up to $15 \mathrm{GHz}$ (HFSS results).

accelerating cell and the modes trapped in the waveguide between the cell and the damper. In all cases the modes have quality factors below 100 and the mode with the quality factor larger than 100 is a TE-like mode that does not completely couple with the dampers but that has a very small transverse impedance. The results of Fig. 10 have been obtained with the PE/PE boundary conditions 2 of Fig. 8(d) but similar results are obtained for other combinations of PE/PH boundaries. We have also performed HFSS simulations considering small differences in the electrical properties of the Ekasic $\mathrm{P}$ (in the range of $\pm 10 \%$ ) and all the previous results are quite similar.

Finally, the distance (h) between the $\mathrm{SiC}$ absorber and the accelerating cell [Fig. 8(d)] has been minimized analyzing the quality factor of the fundamental accelerating mode as a function of the distance itself. The simulation

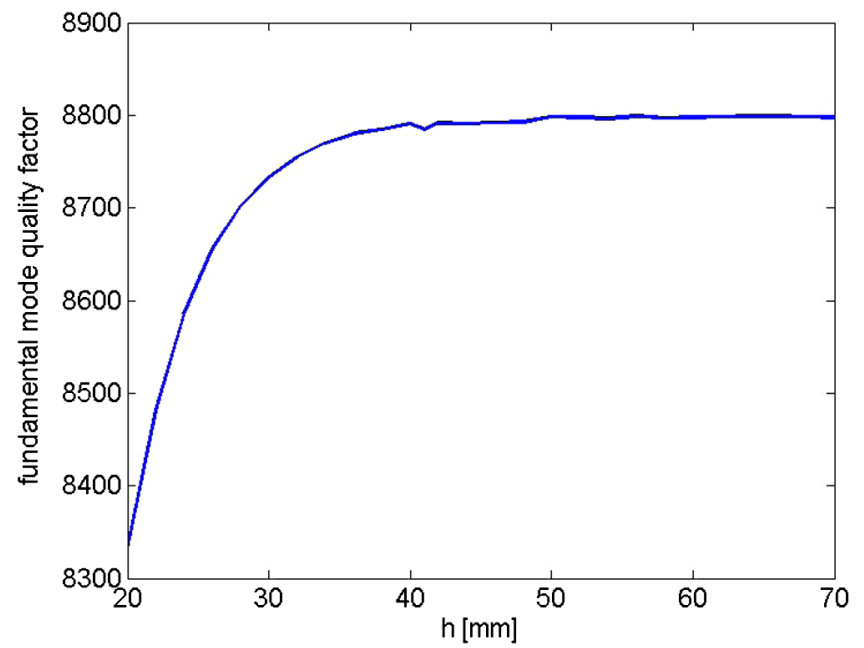

FIG. 11. Quality factor of the fundamental accelerating mode as a function of the distance (h) between the $\mathrm{SiC}$ absorber and the accelerating cell. 
TABLE III. Damped single cell structure main parameters.

\begin{tabular}{lc}
\hline \hline Parameter & Value \\
\hline Working frequency $\left(\mathrm{f}_{\mathrm{rf}}\right)$ & $5.712[\mathrm{GHz}]$ \\
Cell length $(\mathrm{d})$ & $17.495[\mathrm{~mm}]$ \\
Working mode & $\mathrm{TM}_{01}-\mathrm{like}$ \\
Phase advance between cells & $2 \pi / 3$ \\
Series impedance $(\mathrm{Z})$ & $48\left[\mathrm{M} \Omega / \mathrm{m}^{2}\right]$ \\
Field attenuation constant $(\alpha)$ & $0.34[1 / \mathrm{m}]$ \\
Surface peak H field @ $\mathrm{E}_{\mathrm{acc}}=33 \mathrm{MV} / \mathrm{m}$ & $150 \mathrm{kA} / \mathrm{m}$ \\
Surface peak E field @ $\mathrm{E}_{\text {acc }}=33 \mathrm{MV} / \mathrm{m}$ & $68 \mathrm{MV} / \mathrm{m}$ \\
Quality factor $\left(\mathrm{Q}_{0}\right)$ & 8850 \\
Shunt impedance per unit length $(\mathrm{r})$ & $70[\mathrm{M} \Omega / \mathrm{m}]$ \\
Normalized group velocity $\left(\mathrm{v}_{g} / \mathrm{c}\right)$ & 0.02 \\
Iris half aperture $(\mathrm{a})$ & $6.25[\mathrm{~mm}]$ \\
Cell diameter $(\mathrm{b})$ & $19.655[\mathrm{~mm}]$ \\
Iris thickness $(\mathrm{t})$ & $2.5[\mathrm{~mm}]$ \\
Waveguide width $\left(\mathrm{a}_{\text {waveg }}\right)$ & $20[\mathrm{~mm}]$ \\
Waveguide apertures $\left(\mathrm{a}_{\text {damp }}\right)$ & $13[\mathrm{~mm}]$ \\
Distance between the $\mathrm{SiC}$ absorber & $40[\mathrm{~mm}]$ \\
$\quad$ and the accelerating cell $(\mathrm{h})$ & \\
Pulsed heating @ 850 ns rf pulse length & $<6.5^{\circ} \mathrm{C}$ \\
\hline \hline
\end{tabular}

results obtained by HFSS (with the boundary conditions specified in Fig. 8) are given in Fig. 11 and the distance $h$ has been fixed to $40 \mathrm{~mm}$ as a good compromise between final transverse dimensions and perturbation on the accelerating mode.

\section{Final HOM damped single cell parameters}

To take into account the overall response of the structure we have finally simulated a complete 20 -cell structure with the $\mathrm{SiC}$ absorbers [reported in Fig. 6(b)] obtaining the wake potential reported in Fig. 7(a) (red curve). As expected, the decay time is very similar to the perfect matched case. The corresponding transverse impedance is given in Fig. 7(b) and clearly shows that the two cases (with perfect matched ports and with the real dampers) are comparable. The final single cell parameters are given in Table III, with the definitions reported in Appendix A. According to the results discussed in [13], the transverse shape of the irises has been chosen with an elliptical profile (4/3 ellipse axis ratio) in order to reduce the surface peak electric field on the irises themselves. The longitudinal accelerating field profile corresponding to an average value of $\mathrm{E}_{\mathrm{acc}}=33 \mathrm{MV} / \mathrm{m}$ is given in Fig. 12(a) while the related surface electric and magnetic fields are given in Figs. 12(b) and $12(\mathrm{c})$.

\section{E. Iris shaping to obtain a quasiconstant gradient structure}

To calculate the final apertures of the irises of each cell, HFSS simulations have been performed and the main figures of merit (shunt and series impedance per unit length, field attenuation and group velocity, as defined in Appendix A) have been calculated as a function of the iris radius a. The results are summarized in Fig. 13 for an iris thickness $t=2.5 \mathrm{~mm}$ (the iris, as already pointed out, has an elliptical shape).

The iris dimensions, the average accelerating field and the main structure parameters as a function of the longitudinal coordinate $\mathrm{z}$ have then been calculated for three different types of structures [23]: constant impedance (CI), (a)
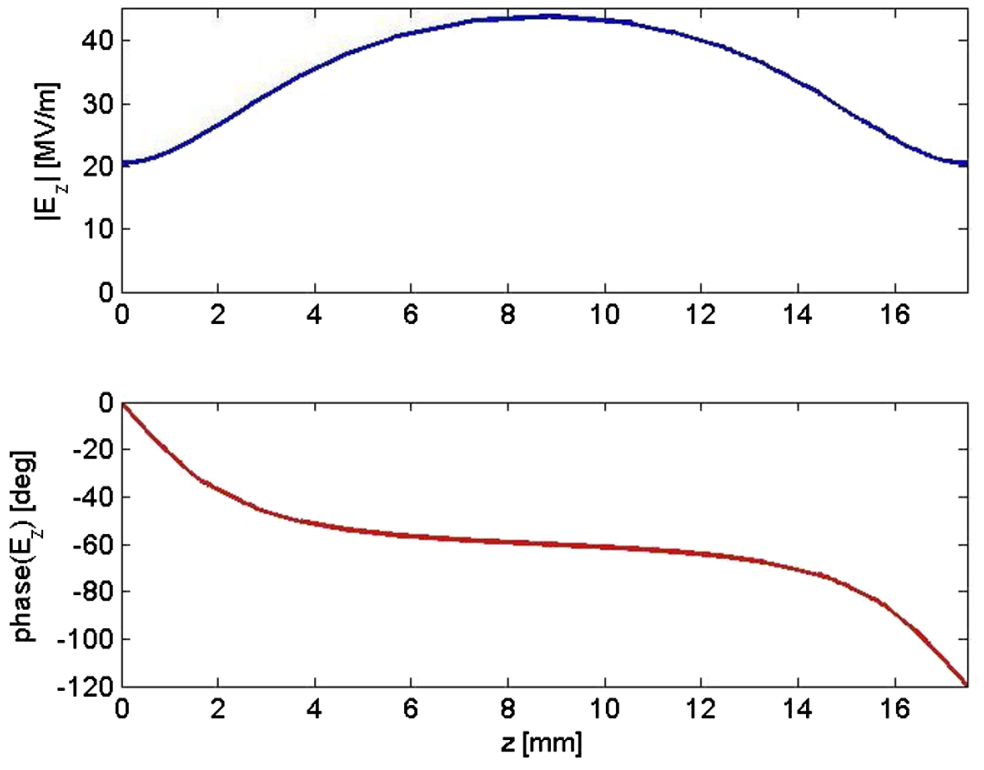
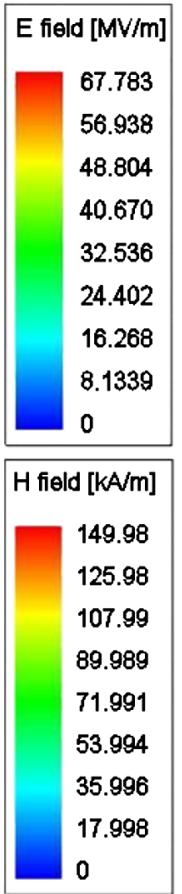

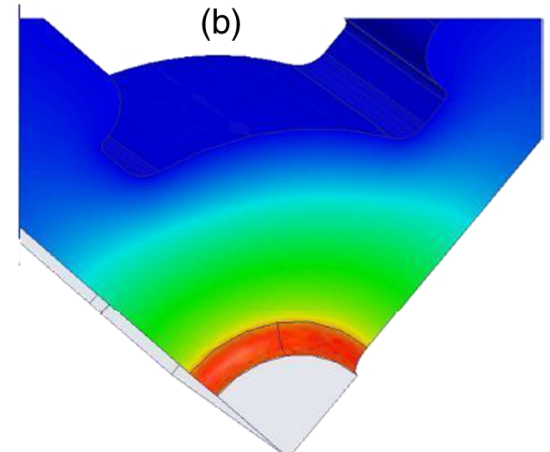

(c)

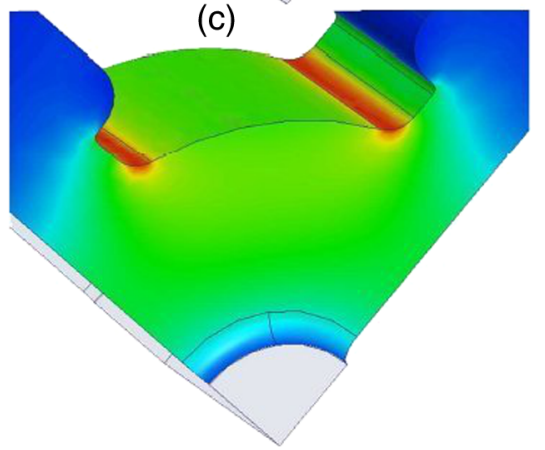

FIG. 12. (a) Longitudinal accelerating field profile at $\mathrm{E}_{\mathrm{acc}}=33 \mathrm{MV} / \mathrm{m}$ and corresponding surface electric (b) and magnetic fields (c). 

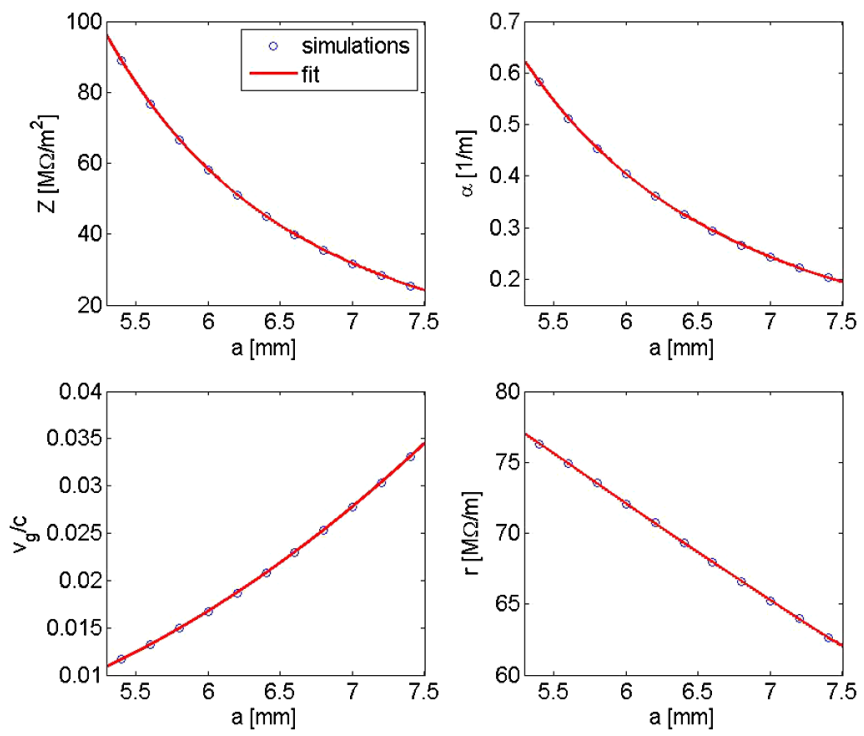

FIG. 13. Single cell parameters as a function of the irises aperture a.

constant gradient $(\mathrm{CG})$ and quasiconstant gradient (quasiCG). CG structures allow having a uniformity of the power dissipation along the section and a lower value of the surface peak electric field for the same energy gain, if the structures are fed by a constant rf input pulse. They have been chosen, as example, for the SLAC S-band $3 \mathrm{~m}$ long structures [24] and for the $\mathrm{C}$ band linac of the SwissFEL $[25,26]$. Nevertheless, if CG structures are fed by a SLED-type rf input pulse [27], they give a not uniform accelerating field and high peak values of the field at the end of the structures, as analyzed and discussed in [13]. In this case CI structures can give better performances in terms of field uniformity [28]. There are also other possibilities as, for example, in the Shintake choke mode $C$-band cavities [3-5,29] where the irises have a linear tapering along the section obtaining a quasiCG structure. We have analyzed the three different cases. The results are given in Fig. 14 and are summarized in Table IV, assuming a perfect uniform input power pulse of $40 \mathrm{MW}$ and a final average accelerating field of $33 \mathrm{MV} / \mathrm{m}$. After this analysis, it has been decided to adopt a quasi-CG structure design with a linear tapering of the irises. In the CI case, in fact, in order to reach an average accelerating field of $33 \mathrm{MV} / \mathrm{m}$, the field in the first cells has to be increased more than $45 \mathrm{MV} / \mathrm{m}$, giving potential problems in terms of breakdown rate. The differences between the $\mathrm{CG}$ and the quasi-CG case are relatively small, but we preferred the second case because the first one requires smaller irises at the end of the structure with a consequent increase of the dipole mode $\mathrm{R} / \mathrm{Q}$, reduction of the pumping speed and beam clearance. The frequency sensitivity with respect to the cell main parameters are given in Table V (they are referred to the central cell of the structure).
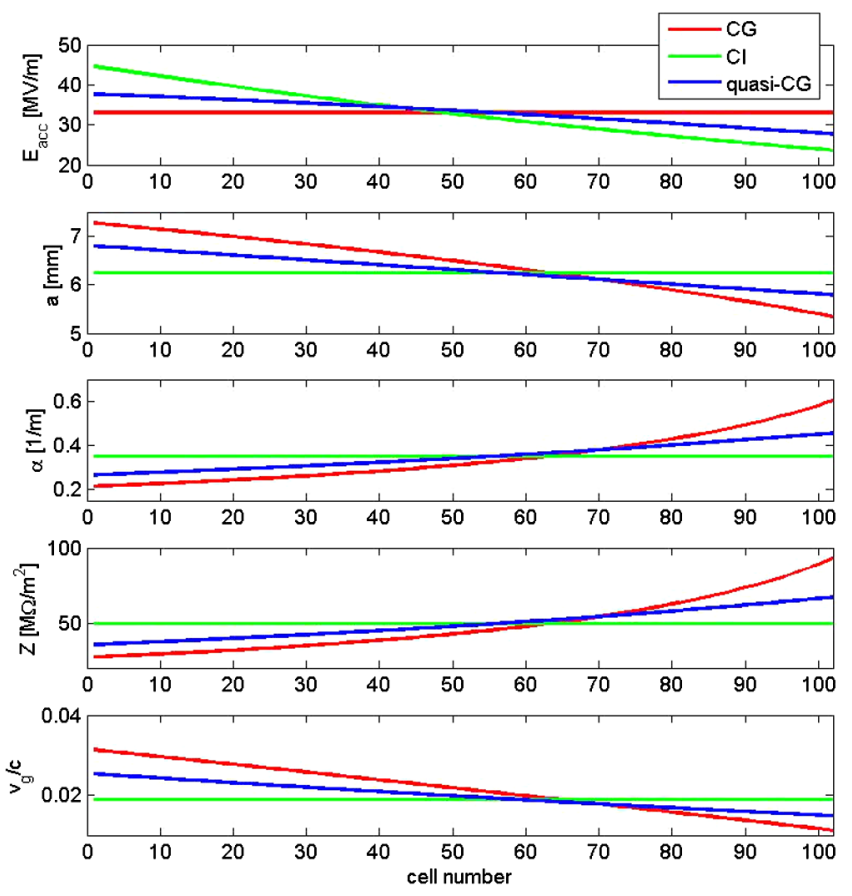

FIG. 14. Average accelerating field, irises dimensions, attenuation constant, series impedance and group velocity as a function of the cell number for different types of structures.

\section{F. Input and output coupler design}

The input and output couplers have been designed with a symmetric feeding and rounded edges to avoid dipole field components and to reduce the pulsed heating on the surfaces [30,31]. The input coupler [shown in Fig. 15(a)] integrates a splitter to allow the symmetric feeding with a single waveguide port, while the output coupler [shown in Fig. 15(b)] has two symmetric outputs connected to two rf loads. The design of both the couplers and of the splitter has been done using HFSS and the technique illustrated in [32].

The input and output coupler cells have been designed with a racetrack shape to suppress the quadrupole field components induced by the presence of the waveguide hole apertures $[33,34]$. It is easy to verify by HFSS simulations that the quadrupole field component in the coupler is completely dominated by the magnetic field asymmetry (see Appendix C). Due to the symmetric feed, these components have an even periodicity with respect to the azimuthal angle. More precisely the azimuthal magnetic field can be developed to first order as follows:

$$
B_{\theta}(r, \vartheta, z) \cong A_{o}(z) r+\sum_{n=1}^{\infty} A_{2 n}(z) \cos (2 n \vartheta) r^{2 n-1}
$$

where the $\mathrm{A}_{2 n}$ components are complex functions and depend on the longitudinal coordinate $\mathrm{z}$. 
TABLE IV. Main parameters of the ELI-NP structures (the final one is the quasi-CG).

\begin{tabular}{lccc}
\hline \hline Structure type & $\mathrm{CG}$ & $\mathrm{CI}$ & Quasi-CG \\
\hline Working frequency $\left(\mathrm{f}_{\mathrm{RF}}\right)$ & & $5.712[\mathrm{GHz}]$ & 102 \\
Number of cells & & $1.8 \mathrm{~m}$ & \\
Structure length & & $\mathrm{TM}_{01}-\mathrm{like}$ & $6.8-5.78 \mathrm{~mm}$ \\
Working mode & $7.27-5.34 \mathrm{~mm}$ & $6.25 \mathrm{~mm}$ & $2 \pi / 3$ \\
Iris half aperture radius & & $40 \mathrm{MW}$ & $37-27 \mathrm{MV} / \mathrm{m}$ \\
Cell phase advance & & $33 \mathrm{MV} / \mathrm{m}$ & $8870-8840$ \\
rf input power & $33 \mathrm{MV} / \mathrm{m}$ & $45-24 \mathrm{MV} / \mathrm{m}$ & $67-74 \mathrm{M} \Omega / \mathrm{m}$ \\
Average accelerating field & $8900-8830$ & 8850 & $0.025-0.015$ \\
Accelerating field input-output & $63-77 \mathrm{M} \Omega / \mathrm{m}$ & $70 \mathrm{M} \Omega / \mathrm{m}$ & $313 \mathrm{~ns}$ \\
Average quality factor & $0.031-0.011$ & 0.019 & $0.29 \cdot \mathrm{Pin}$ \\
Shunt impedance & 308 & 314 & \\
group velocity $\left(\mathrm{v}_{g} / \mathrm{c}\right)$ & $0.29 \cdot \mathrm{Pin}$ & $0.28 \cdot \mathrm{Pin}$ & $512 \mathrm{~ns}$ \\
Filling time & & $100 \mathrm{~Hz}$ & $<8^{\circ} \mathrm{C}$ \\
Output power & & $<12^{\circ} \mathrm{C}$ & $<21^{\circ} \mathrm{C}$ \\
Pulse duration for beam $\left(\tau_{\mathrm{BEAM}}\right)$ & $2.3 \mathrm{~kW}$ & $30^{\circ} \mathrm{C}$ \\
Repetition rate $\left(\mathrm{f}_{\text {rep }}\right)$ & $<6.5^{\circ} \mathrm{C}$ & & \\
Pulsed heating accelerating cells & & & \\
Pulsed heating input coupler & & & \\
Average dissipated power & & & \\
Working temperature & & &
\end{tabular}

TABLE V. Frequency $(\mathrm{df} / \mathrm{dx})$ and phase advance per cell $(\mathrm{d} \phi / \mathrm{dx})$ sensitivities with respect to the cell main geometrical parameters.

\begin{tabular}{lcc}
\hline \hline Parameter & $\begin{array}{c}\text { Sensitivity }(\mathrm{df} / \mathrm{dx}) \\
{[\mathrm{kHz} / \mu \mathrm{m}]}\end{array}$ & $\begin{array}{c}\text { Sensitivity }(\mathrm{d} \phi / \mathrm{dx}) \\
{[\mathrm{deg} / \mu \mathrm{m}]}\end{array}$ \\
\hline Radius of the cell (b) & -300 & 0.31 \\
Cell iris (a) & 70 & -0.074 \\
Cell length (d) & -10 & 0.011 \\
Iris thickness (t) & 20 & -0.021 \\
\hline \hline
\end{tabular}

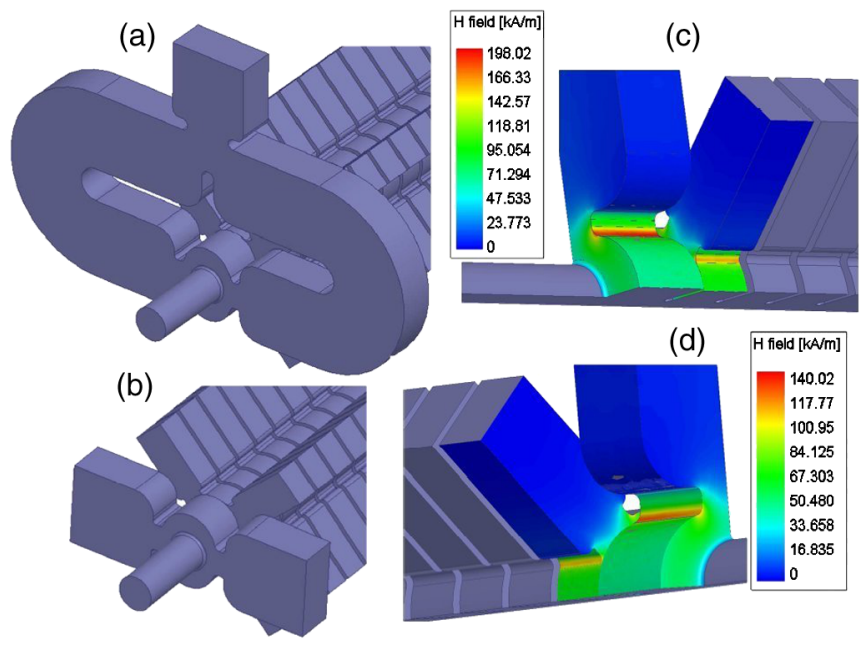

FIG. 15. (a) input coupler; (b) output coupler; (c) surface magnetic field at the input coupler corresponding to $\mathrm{E}_{\mathrm{acc}}=33 \mathrm{MV} / \mathrm{m}$; (c) surface magnetic field at the output coupler corresponding to $\mathrm{E}_{\mathrm{acc}}=33 \mathrm{MV} / \mathrm{m}$.
The quadrupolar component is the component associated to the term with $n=1$ and the gradient of the quadrupole component is exactly the term $\mathrm{A}_{2}$.

Figure 16 shows, as an example, the magnitude of the azimuthal magnetic field in the center of the input coupler for three different circle radii and for one quarter of the structure $(\theta=0$ is below the coupling hole). The calculation has been performed for an input power of $40 \mathrm{MW}$. The field profile is mainly driven by the quadrupolar component, but shows also other high order components that are more evident for a larger radii.

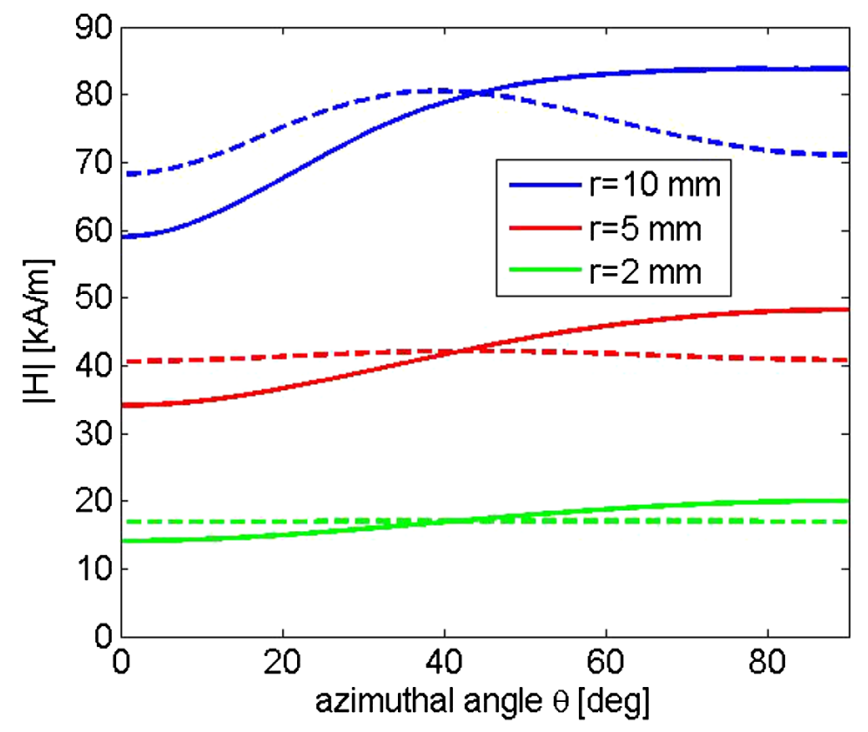

FIG. 16. Azimuthal magnetic field in the center of the input coupler calculated for three different circle radii (continuous lines: without racetrack; dashed lines: with racetrack). 


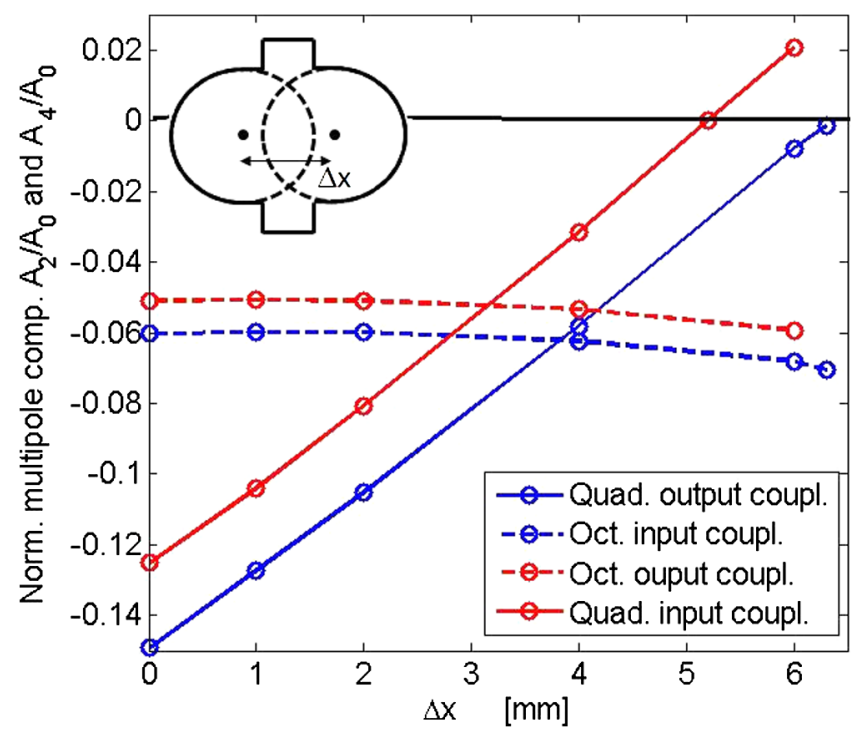

FIG. 17. Multipole magnetic field components in the center of the input/output coupler. The components have been calculated considering the absolute value of the magnetic field $\left|\mathrm{B}_{\theta}\right|$ and developing it according to Eq. (3).

The amplitudes of the quadrupole and octupole field components in the center of the input and output couplers, as a function of the racetrack thickness $\Delta \mathrm{x}$, are given in Fig. 17. The components have been calculated considering the absolute value of the magnetic field and developing it according to Eq. (3). In principle it is possible to calculate the multipolar field components as a function of the longitudinal coordinate (z) along the couplers by calculating the Fourier series components in different arcs at different longitudinal positions. It is, nevertheless, quite easy to verify that the amplitude of this quadrupole field component is maximum in the center of the coupler itself and that the minimization of this component in that position allows one to minimize the overall quadrupole component integrated along the whole coupler. A detailed discussion of the last point is given in Appendix C. From Fig. 17 it is also easy to note that the octupole component is practically unperturbed by the racetrack geometry. The final coupler racetrack dimensions have been chosen equal to $\Delta \mathrm{x}=$ $6.3 \mathrm{~mm}$ and $\Delta \mathrm{x}=5.2 \mathrm{~mm}$ for the input and output coupler respectively, and the azimuthal magnetic field in the center of the input coupler with the racetrack geometry is still given in Fig. 16.

With HFSS we have also simulated the full structure with the final input and output couplers. The result of the longitudinal electric field profile along the structure is given in Fig. 18 while the surface magnetic field on the couplers is shown in Fig. 15, the corresponding pulsed heating in the input coupler is below $21^{\circ} \mathrm{C}$. The residual small field unflatness (and the corresponding phase advance per cell within a few degrees with respect to the nominal one) is due to a small reflection on the output coupler and structure cells caused by numerical errors and a

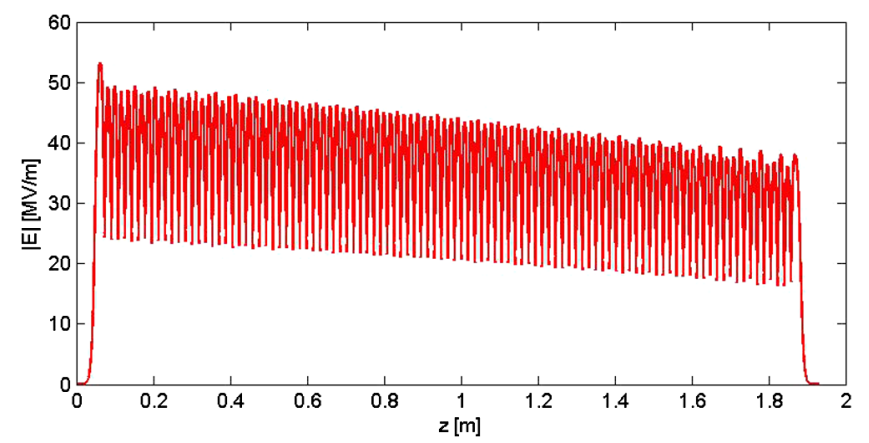

FIG. 18. Longitudinal electric field profile along the whole structure simulated by HFSS.

very large number of simulated tetrahedra (more than $10^{6}$ ). In any case all fluctuations are within the tunability of the structure [35]. The simulated final reflection coefficient at the input port is below $-30 \mathrm{~dB}$.

\section{THERMAL AND VACUUM ANALYSIS}

A straightforward thermomechanical analysis and a totally coupled loop analysis [36] have been performed with ANSYS [17] on the whole structure. Through these two complementary analyses, it was possible to check the cooling efficiency, the mechanical behavior (displacement due to thermal gradient in the copper body) and related detuning, under the $100 \mathrm{~Hz}$ operation with $2.3 \mathrm{~kW}$ average dissipated power.

Also vacuum calculations have been performed to evaluate the vacuum pressure along the structure itself [37] due to the presence of the $\mathrm{SiC}$ absorbers distributed along the structure.

\section{A. Thermomechanical analysis}

For the first analysis, the electromagnetic (EM) field has been imported from simulation (HFSS ANSYS module), inside a thermal simulation module (ANSYS steady-state thermal), as heat fux on the internal cavity surfaces $\left(\mathrm{W} / \mathrm{m}^{2}\right)$. At this stage, the rough geometry coming from the EM model has been refined, and the cooling channels have been 3D-modeled pursuant mechanical drawings of the structure. Inside channels, line bodies have been defined and then meshed with fluid elements (through a dedicated APDL command script) in order to simulate the real convective heat exchange with copper. Convection with surrounding air has been considered in the same module. The results of the analysis were the coolant heating (confirming the dissipated power) and the temperature distribution of the structure. The second analysis (totally coupled) closed the loop: the static-structural ANSYS module has been added to simulation in order to calculate the structure deformation related to the temperature distribution from the previous thermal module, and mechanically constrained to simulate operative support conditions 


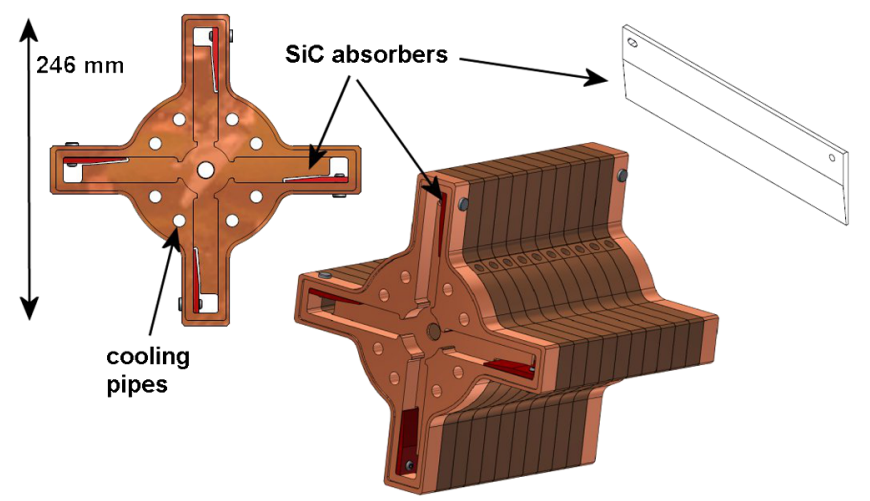

FIG. 19. Final mechanical drawing of the single cell and of the 12-cell module.

(the deformation calculated must be as much homothetic as possible, in order to minimize structure detuning). Once the simulation has run, the outcoming deformed mesh has been sent back to HFSS in order to calculate detuning and close the "loop." The final mechanical drawing of the single cell and of the 12-cell module is depicted in Fig. 19. Each structure presents 12 longitudinal cooling channels (ID $10 \mathrm{~mm}$ ): 2 for the input coupler, 2 for the output coupler and 8 for the structure obtained by brazing of single cells. Simulating a total water flow of 66 litres/min, the

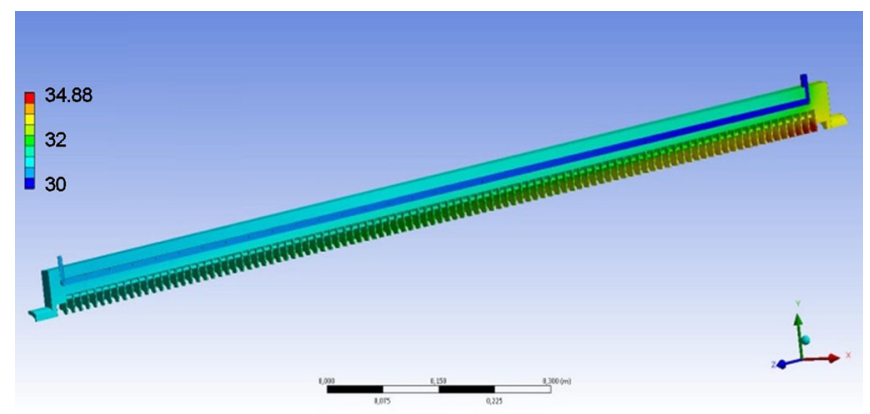

FIG. 20. Temperature distribution along the $C$-band structure. calculated power dissipation was about $2.3 \mathrm{~kW}$ in water, $6 \mathrm{~W}$ in air (water temperature $30^{\circ} \mathrm{C}$, air temperature $22^{\circ} \mathrm{C}$ ). The temperature distribution calculated $\left(\Delta \mathrm{T}_{\max } \approx 5^{\circ}\right)$, represented in Fig. 20, develops mainly along the longitudinal axis direction (i.e. on more than one hundred cells). In the single resonant cell in fact the temperature distribution was almost uniform, involving a maximum mechanical deformation of a few $\mu \mathrm{m}$ (largely allowable). The deformations of the structure in the radial direction are given in Fig. 21 while, in the longitudinal direction, the calculated deformation of the overall structure is less than $55 \mu \mathrm{m}$. According to the sensitivities reported in Table $\mathrm{V}$ the maximum cell to cell frequency shifts are below $5 \mathrm{kHz}$ and the corresponding cell to cell phase advance variations are less than $0.005 \mathrm{deg}$.

\section{B. Vacuum analysis}

Two pumping units are foreseen in the structure, one at the entrance and one at the end. The pressure profile along the axis of the structure is given in Fig. 22 for two different values of the specific outgassing rate of the copper and of the $\mathrm{SiC}$ absorbers. In the first case (upper plot) a conser-

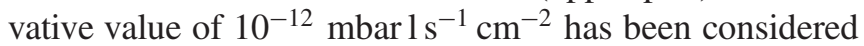
for both copper and $\mathrm{SiC}$ while in the second case (bottom plot) a value of $2 \times 10^{-14} \mathrm{mbarl} \mathrm{s}^{-1} \mathrm{~cm}^{-2}$ and a value of $5 \times 10^{-13} \mathrm{mbarl} \mathrm{s}^{-1} \mathrm{~cm}^{-2}$ have been considered for copper and $\mathrm{SiC}$ respectively. The first values are related to materials without bakeout treatment while the second case to a particular thermal procedure, called vacuum firing, which consists in keeping the structure at 550 Celsius degrees in a vacuum oven for a few days at a pressure lower than $10^{-7}$ mbar. The two plots related to the worst and best specific outgassing rate values give two pressure profiles in which the structure will operate. It is quite easy to verify that, even in the worst case, the pressure in the center of the structure is below $8 \times 10^{-8}$ mbar that is absolutely compatible for a high gradient operation.
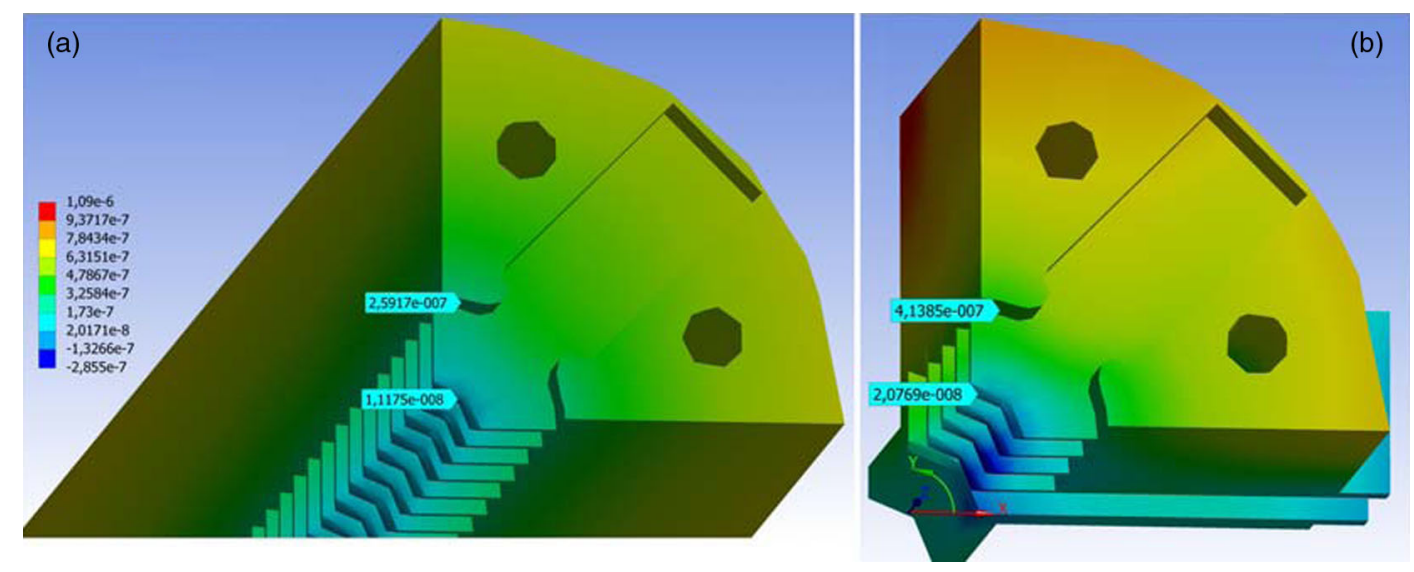

FIG. 21. Radial deformations: (a) last part of the structure (cell number 95); (b) first part of the structure (cell number 4). The probes indicate the deformations on the outer diameter of the cell and on the irises. 


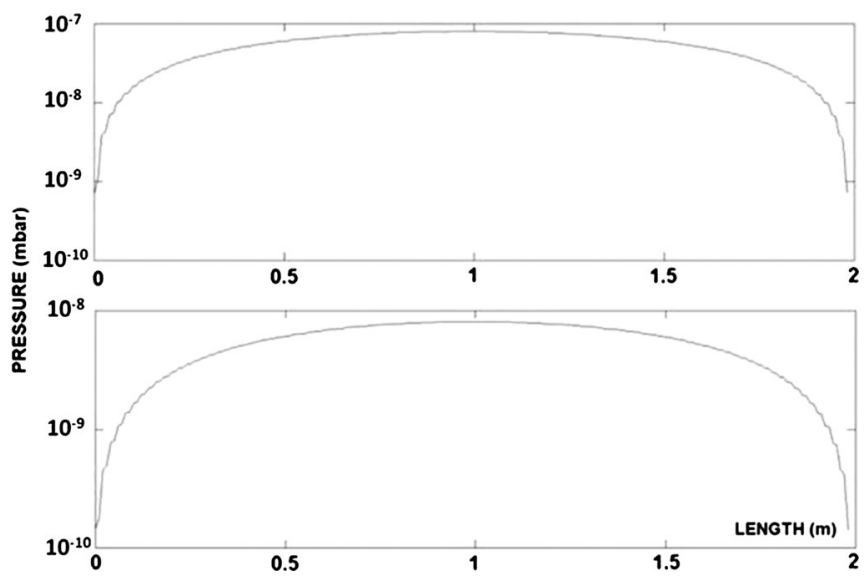

FIG. 22. Pressure profile along the axis of the structure for two different values of the specific outgassing rate.

\section{BEAM LOADING COMPENSATION}

Because of the multibunch operation, also the beam loading effects have been studied. The beam loading decreases the accelerating field gradient in the structure since the effective field is the superposition of the external rf field and beam induced wakefield. A complete discussion on beam loading in TW structures and possible compensation techniques can be found elsewhere in several published papers [38-44]. When a single bunch passes through a TW structure it leaves part of its energy exciting the fundamental mode with a decelerating phase. The profile of the accelerating field perturbation travels forward along the structure with a local velocity equal to the group velocity of the structure, while the phase of the wave travels with the beam velocity $(\sim c)$. The total accelerating field probed by a subsequent bunch is the superposition of the propagating perturbation generated by the leading bunch and the external rf field. This simplified treatment neglects the dispersive effects related to the fact that the beam excites the field at all frequencies of the passband accelerating mode. All field components propagate into the structure with different group and phase velocities. A complete treatment has been done for other structures [42-44] and demonstrated that this simplified approach describes with an acceptable approximation the whole beam loading process.

The described mechanism is sketched in Fig. 23. Each bunch generates a traveling beam loading field profile [Fig. 23(a)] and, if the bunch train duration is longer than the structure filling time, the beam loading field reaches a steady state condition [Fig. 23(b)].

For the ELI-NP nominal beam the energy spread generated along the train by beam loading effects is given in Fig. 24 (blue and red dots). The figure shows both the transient beam loading and the steady state regime (red dots). The steady state is reached since the bunch train is longer than the structure filling time. Clearly, if not compensated, the beam loading produces a bunch-to-bunch energy spread along the train well beyond the machine specifications. There are quite a few techniques to compensate these unwanted effects; some of them are based on a proper modulation of the amplitude and phase of the rf input power [40-46], others are based on a proper choice of the beam injection time, with respect to the energy filling of the structures [44].

In our case we propose to use an amplitude modulation of the input power acting on the low level rf system driving the klystron. The concept is quite simple: prior to the beam injection, the structure has to be filled with a proper shaped input pulse, to preload an accelerating field profile equal to the steady state beam loading one. Therefore, since the injection of the very first bunch the beam "experiences" exactly the steady state beam loading field profile. The details of the analysis are given in Appendix B. In Fig. 24 we report the final input pulse that allows avoiding the beam loading transient along the train, and the expected bunch energy spread with and without compensation. The required energy spread bunch by bunch should be below $0.1 \%$ [1].

(a)

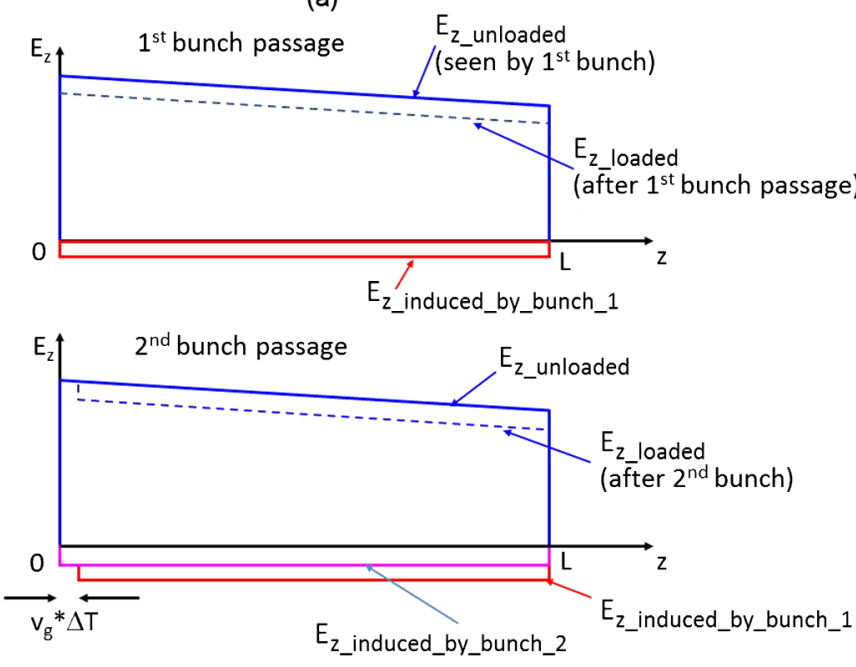

(b)

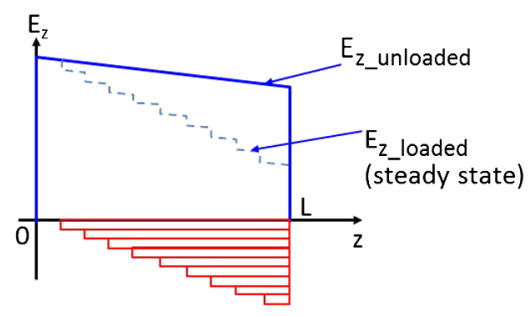

FIG. 23. Schematic view of the beam loading process in TW structures: (a) traveling beam loading field profile generated by two bunches; (b) steady state condition. 


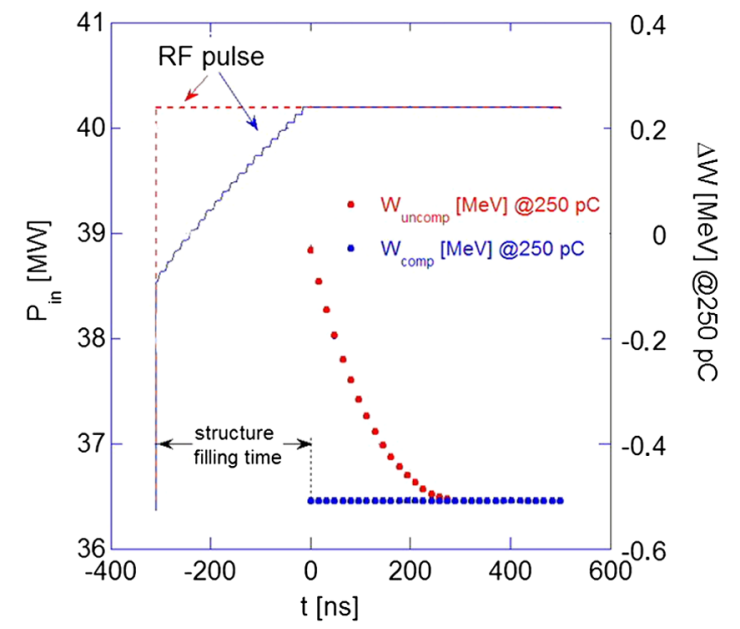

FIG. 24. rf pulse shape without shaping (red dashed curve, left scale) and with shaping for beam loading compensation (blue curve, left scale) and bunch energy distribution (right scale) with (blue dots) and without (red dots) compensation.

The shaping of the rf pulse as given in Fig. 24 is ideal because, in reality, one has to consider the distortions due to the finite bandwidth of the rf system and klystron. Also the dispersive effects of the TW structure [47] will affect the final accelerated gradient sampled by bunches.

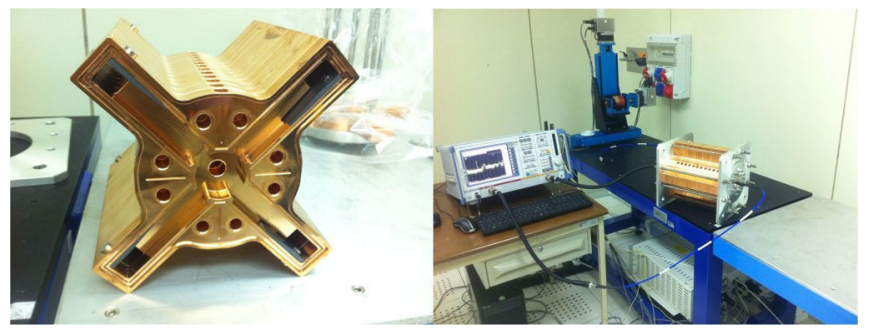

FIG. 25. Picture of a 12-cell module (left); measurements setup (right).

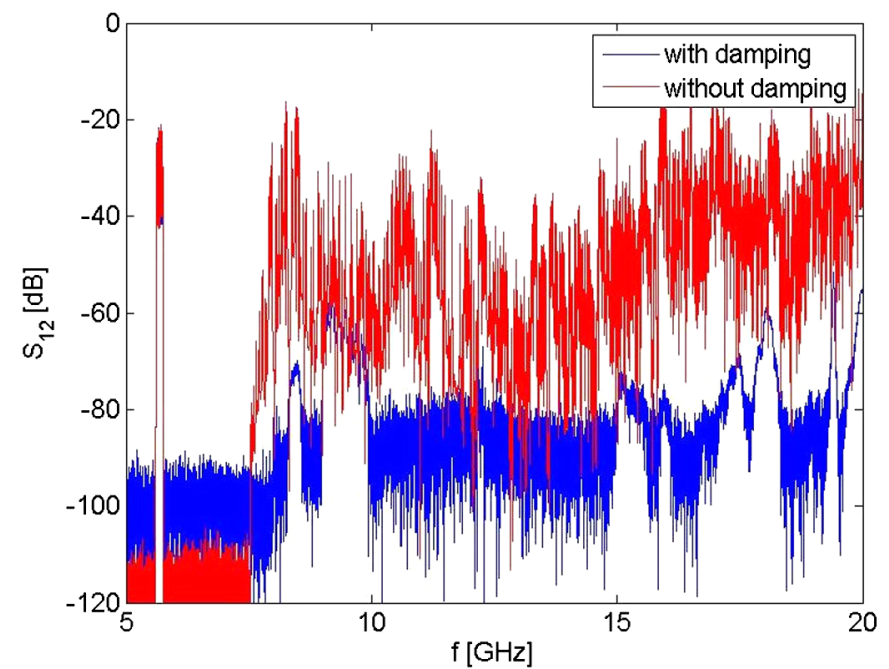

The modeling of all these effects is beyond the scope of the present paper and requires a set of experimental measurements performed on the final rf power system, and a simulation of the TW structure at different frequencies to take into account also the dispersive effects. On this basis, a more realistic and accurate rf pulse profile could can be computed.

\section{PROTOTYPES AND RF MEASUREMENTS ON THE EFFECTIVENESS OF THE SIC ABSORBERS}

The realization and the final test of the cavities will be the subject of a dedicated paper. Here we would like to remark that an intense prototyping activity has been carried out to set up and optimize the final realization process $[16,48]$. A picture of a single 12-cell module is given, as an example, in Fig. 25. The cells have been machined in an private company [49] and have been brazed at LNF-INFN with Palcusil 10 alloy [50] at 850 degrees in a vacuum furnace. An rf test at low power has been performed on this module with and without the $\mathrm{SiC}$ absorbers. The results are given in Fig. 26 where the transmission coefficient between two antennas coupled with the structure modes is reported. The measurements show the effectiveness of the $\mathrm{SiC}$ absorbers since the HOMs disappear after their insertion in the structure (Fig. 26(a)). The remaining modes with quality factors of a few hundred are TE-like modes that have a negligible transverse impedance. On the other hand the accelerating modes of the first band are unperturbed as is clearly visible in Fig. 26(b).

\section{ACKNOWLEDGMENTS}

First of all we would like to thank A. Grudiev and G. De Michele for their help in the methodology of the design of the damped structures and rf measurements of the $\mathrm{SiC}$

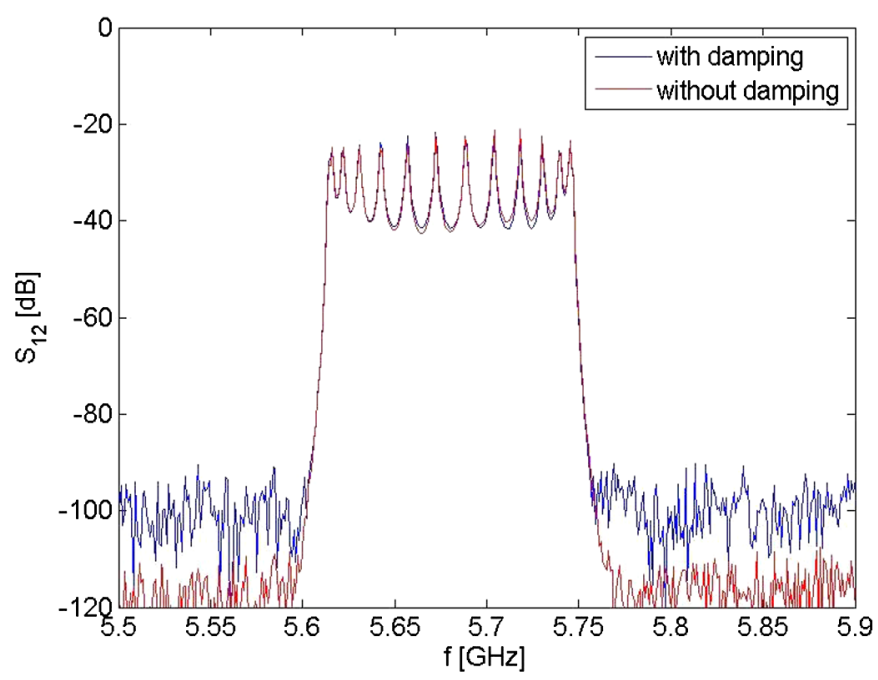

FIG. 26. Transmission coefficient between two antennas coupled to a 12-cell module with and without the absorbers (left); detail of the transmission coefficient corresponding to the bandwidth of the accelerating mode (right). 
absorbers. We would like also to thank R. Di Raddo and P. Chimenti for the technical support and G. Riddone and her collaborators for the helpful suggestions in the mechanical design. Finally we would like to thank I. Syratchev, V. Dolgashev, W. Wuensch, L. Serafini, O. Frasciello, A. Variola, A Ghigo, C. Vaccarezza, R. Boni and the COMEB company for the helpful scientific and technical suggestions and discussions.

\section{APPENDIX A: CALCULATION OF FIGURES OF MERIT OF THE $C$-BAND SINGLE CELL}

The definitions we adopted for the main single cell figures of merit are given below:

$$
\begin{aligned}
Z & =\frac{E_{\text {acc }}^{2}}{P_{\text {in }}} \quad \text { series impedance }\left[\frac{\Omega}{m^{2}}\right] \\
\alpha & =\frac{p_{\text {diss }}}{2 P_{\text {in }}} \quad \text { field attenuation constant }\left[\frac{1}{m}\right] \\
v_{g} & =\frac{P_{\text {in }}}{w} \quad \text { group velocity }\left[\frac{m}{s}\right] \\
r & =\frac{E_{\text {acc }}^{2}}{p_{\text {diss }}}=\frac{Z}{2 \alpha} \quad \text { shunt impedance per unit length }\left[\frac{\Omega}{m}\right] \\
Q & =\omega_{R F} \frac{w}{p_{\text {diss }}}=\omega_{R F} \frac{1}{2 \alpha v_{g}} \quad \text { quality factor, } \quad(\mathrm{A} 1)
\end{aligned}
$$

Where

$E_{z} \quad$ is the complex accelereting field on axis $[\mathrm{V} / m]$

$E_{\mathrm{acc}}=\frac{V_{z}}{d}=\frac{\left|\int_{0}^{d} E_{z} \cdot e^{j \omega_{R F^{\frac{z}{c}}}} d z\right|}{d} \quad$ is the average accelerating field $(d$ is the cell length $)[\mathrm{V} / m]$

$P_{\text {in }}=\int_{S} \frac{1}{2} \operatorname{Re}\left(\underline{E} \times \underline{H}^{*}\right) \cdot \hat{z} d S \quad$ is the average input power $(S$ is the cell transverse section $)[W]$

$P_{\text {diss }}=\frac{1}{2} R_{s} \int_{S_{\mathrm{ext}}}\left|H_{\mathrm{tan}}\right|^{2} d S_{\mathrm{ext}} \quad$ is the average dissipated power in the cell $\left(S_{\mathrm{ext}}\right.$ is the cell surface $)[W]$

$p_{\text {diss }}=\frac{P_{\text {diss }}}{d} \quad$ is the average dissipated power per unit length $[\mathrm{W} / m]$

$W=\frac{1}{4} \mu_{0} \int_{V} \underline{H} \cdot \underline{H}^{*} d V+\frac{1}{4} \varepsilon_{0} \int_{V} \underline{E} \cdot \underline{E}^{*} d V \quad$ is the stored energy in the cell ( $V$ is the cell volume $)[J]$ $w=\frac{W}{d} \quad$ is the average stored energy per unit length $[\mathbf{J} / m]$.

\section{APPENDIX B: BEAM LOADING COMPENSATION IN ELI-NP $C$-BAND STRUCTURES}

The main parameters of the structure available from the EM simulation, which have been used to evaluate the beam loading contribution, are the linear field attenuation $\alpha$, the group velocity $v_{g}$ and the series impedance $Z$ for each cell.

The linear attenuation has been fitted with a third degree polynomial:

$$
\alpha(z)=\alpha_{0}+\alpha_{1} z+\alpha_{2} z^{2}+\alpha_{3} z^{3}
$$

and its trend as a function of the structure longitudinal coordinate $(\mathrm{z})$ is shown in Fig. 27.

Then, the quality factor $\left(Q=\frac{\omega_{R F}}{2 \alpha v_{q}}\right)$ has been computed for each cell and reported in Fig. 28.

Since its variation along the structure is rather small (mean $=8860.1 ;$ standard deviation $=8.3$ ), the quality

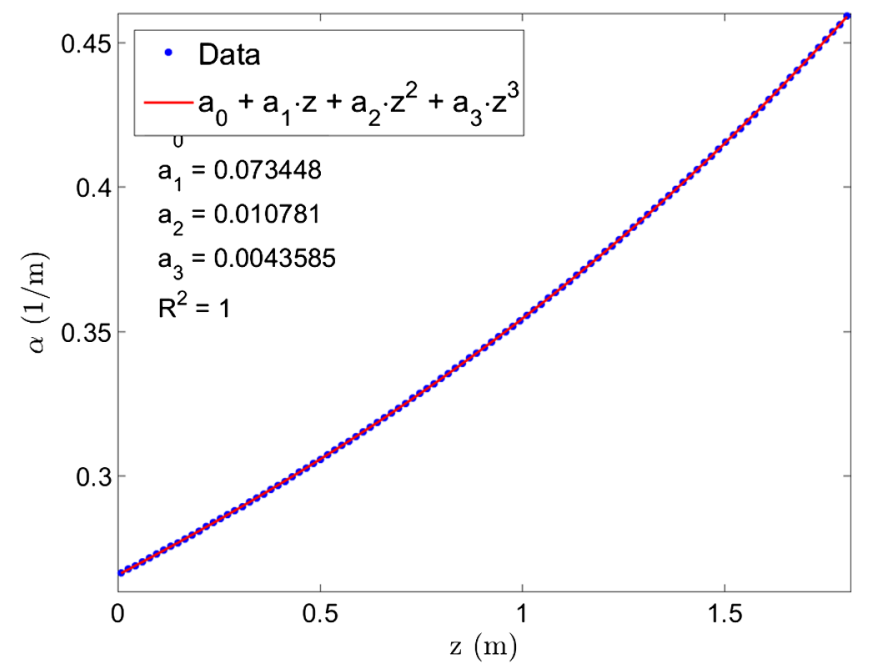

FIG. 27. Field attenuation per unit length within the structure (blue); third order polynomial fit (red). 


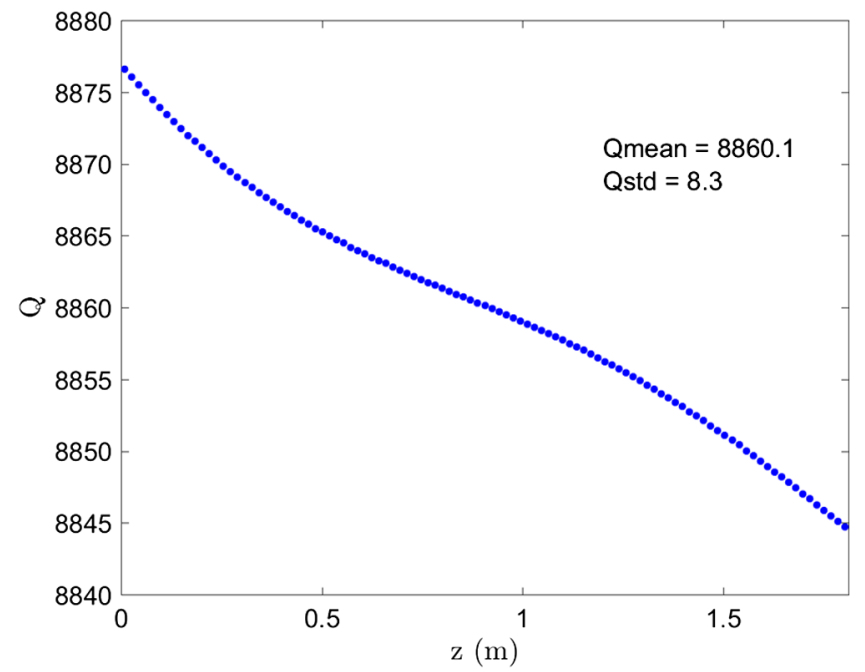

FIG. 28. Quality factor as a function of $\mathrm{z}$ (mean $=8860.1$, sigma $=8.3$ ).

factor has been considered to be constant and equal to its mean value. Then, a direct dependence between $\alpha$ and $\mathrm{v}_{g}$ is established:

$$
v_{g}(z)=\frac{\omega_{R F}}{2 Q_{\mathrm{avg}} \alpha(z)} .
$$

The pulse front edge travels within the structure with a nonuniform velocity, with $f(t)$ its equation of motion:

$$
\begin{aligned}
v_{g}(z) & =\frac{d z}{d t}=\frac{\omega_{R F}}{2 Q_{\mathrm{avg}} \alpha(z)} \rightarrow \alpha(z) d z \\
& =\frac{\omega_{R F}}{2 Q_{\mathrm{avg}}} d t \rightarrow t=f^{-1}(z)=\frac{2 Q_{\mathrm{avg}}}{\omega_{R F}} \sum_{0}^{3} \frac{\alpha_{k}}{k+1} z^{k+1} .
\end{aligned}
$$

Hence, $f^{-1}(z)$ can be expressed as a function of $\mathrm{a}_{k}$ parameters only and it has been plotted in Fig. 29.

Also the function $z=f(t)$ has been calculated, and fitted with a second order polynomial, as shown in Fig. 30, for reasons that will be explained in the following.

The rf power and the accelerating field in the $C$-band section could be derived from:

$$
\begin{aligned}
\frac{d P}{d z} & =-2 \alpha(z) P \rightarrow \frac{d P}{P}=-2 \alpha(z) d z \rightarrow \ln \left(\frac{P}{P_{0}}\right) \\
& =-2 \sum_{0}^{3} \frac{\alpha_{k}}{k+1} z^{k+1}=-\frac{\omega_{R F}}{Q_{\mathrm{avg}}} f^{-1}(z) \\
P & =P_{0} e^{-2 \sum \frac{\alpha_{k}}{k+1} z^{k+1}}=P_{0} e^{-\frac{\omega_{R F}}{Q_{\text {avg }}} f^{-1}(z)} \\
E(z) & =\sqrt{Z(z) P_{0} e^{-2 \sum \frac{\alpha_{k}}{k+1} z^{k+1}}}=\sqrt{Z(z) P_{0} e^{-\frac{\omega_{R F}}{Q_{a v g}} f^{-1}(z)}} .
\end{aligned}
$$

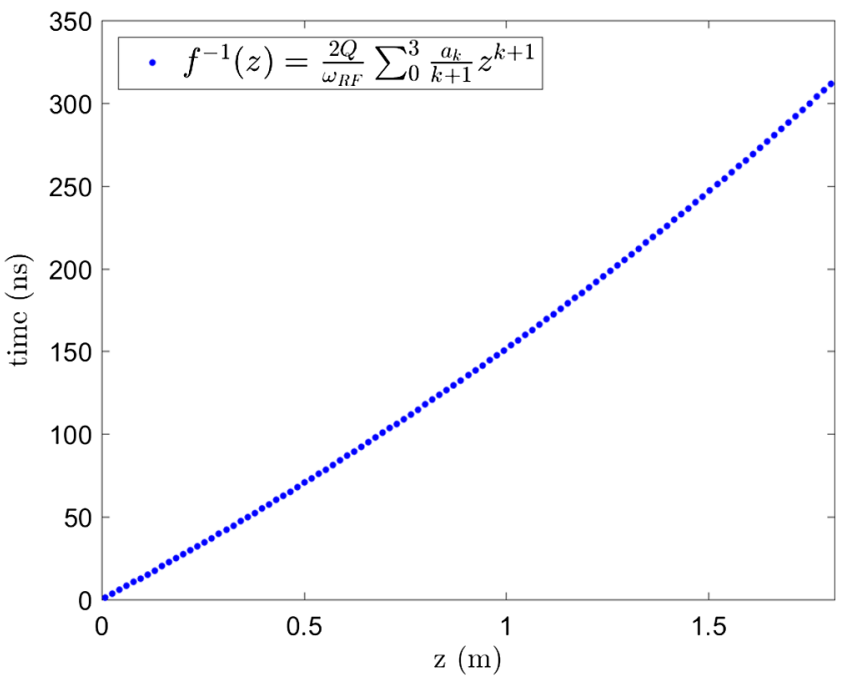

FIG. 29. Inverse equation of motion of the pulse front edge $f^{-1}(z)$.

These two quantities have been normalized to their peak values and reported in Fig. 31.

The function $\eta(z)=E(z) / E_{0}$ is the linear form factor of the field along the structure (i.e. the profile of the blue curve in Fig. 31). Thus, if the field value at a given position $z_{1}$ is $E\left(z_{1}\right)=E_{1}$, when the wave reaches $z_{2}>z_{1}$ the field value will be:

$$
E\left(z_{2}\right)=E_{2}=E_{1} \frac{\eta\left(z_{2}\right)}{\eta\left(z_{1}\right)} .
$$

The electric field generated by a pointlike charge traveling within the structure (normalized to its charge) can be determined by:

$$
W(z)=\frac{1}{2} \omega_{R F} \frac{r}{Q}=\alpha v_{g} r
$$

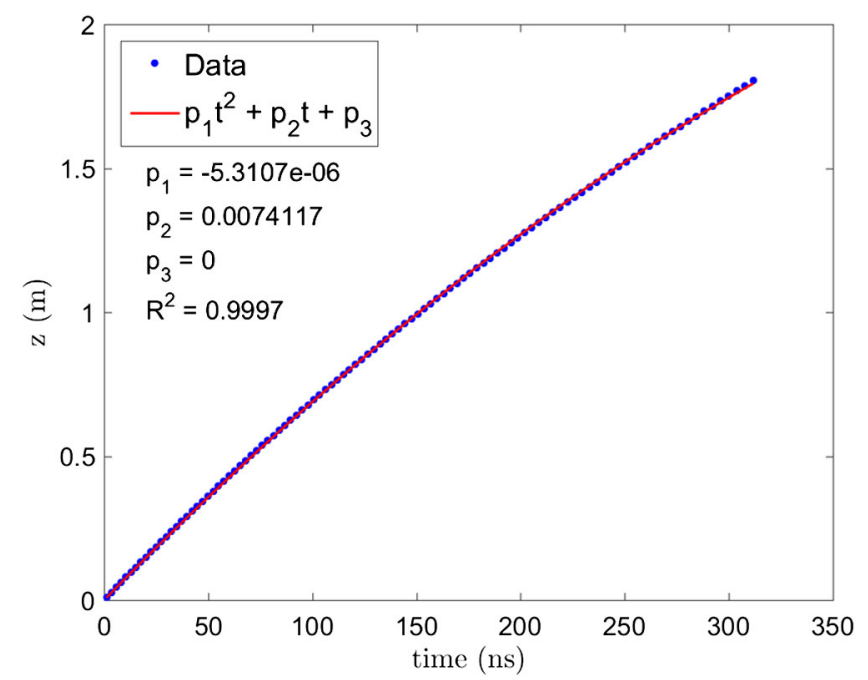

FIG. 30. Equation of motion of the pulse front edge $\boldsymbol{f}(\boldsymbol{t})$ calculated values (blue), second order polynomial fit (red). 


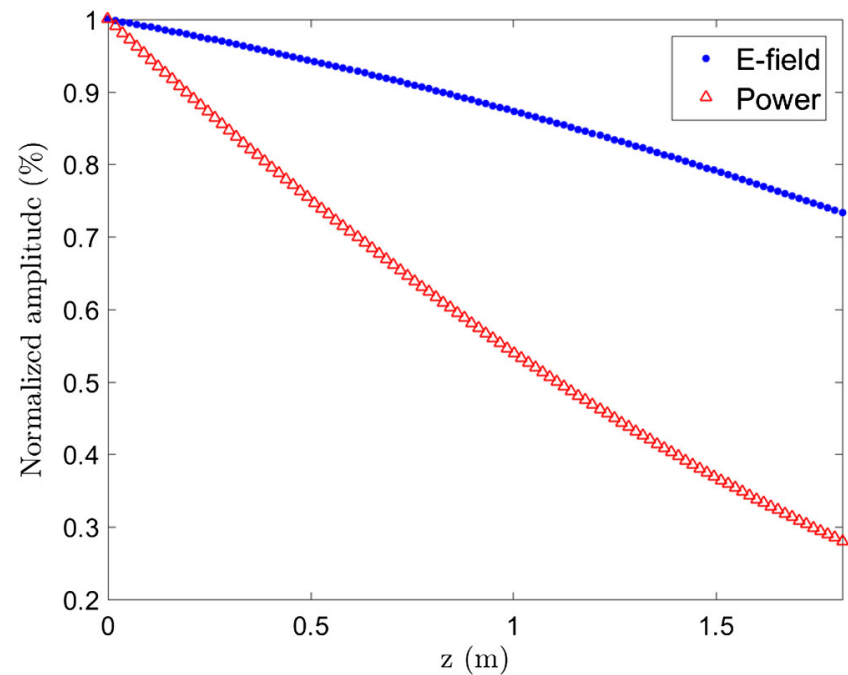

FIG. 31. Normalized accelerating field (blue) and rf power (red) in the structure.

with $r=\frac{E^{2}}{\left|\frac{d P}{d Z}\right|}=\frac{Z}{2 \alpha}$ the shunt impedance of each cell of the section. If we suppose the bunch to be a pointlike charge, the wake potential as a function of $\mathrm{z}$ can be calculated, as shown in Fig. 32.

The function $W(z)$ represents the normalized electric field in the structure just after the passage of a pointlike bunch, i.e. the field profile at $t=0$ or $W(z, t=0)$. The wake travels within the structure according to the same nonuniform equation of motion $f(z)$, shown in Fig. 29, and is attenuated as $\eta(z)$. After a certain amount of time $t>0$, it becomes:

$$
W(z, t>0)=W\left(z^{\prime}, 0\right) \cdot \frac{\eta(z)}{\eta\left(z^{\prime}\right)} \cdot H\left(z^{\prime}\right)
$$

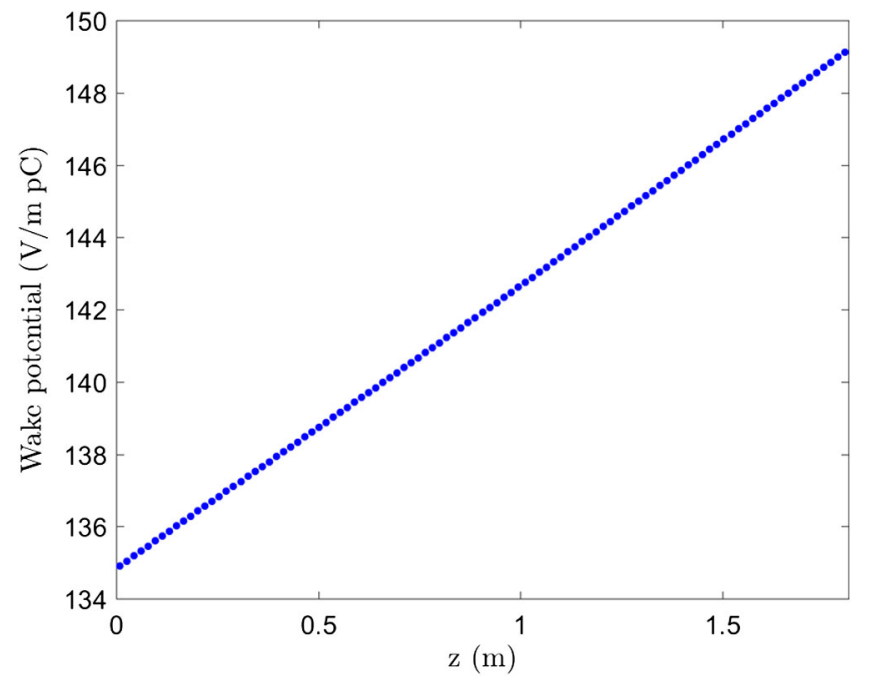

FIG. 32. Normalized wake potential generated by a charge of $1 \mathrm{pC}$ in the structure $\mathrm{W}(\mathrm{z})$. where $z^{\prime}(z, t)$ is the position at time $t=0$ of the wavelet corresponding to position $z$ at time $t>0$. The use of the Heaviside function $\mathrm{H}\left(\mathrm{z}^{\prime}\right)$ is necessary to confine the wake within the structure. The value of $z^{\prime}(z, t)$ can be calculated using the aforementioned equation of motion $f(t)$ and its inverse function $f^{-1}(z)$ :

$$
z^{\prime}=f\left[f^{-1}(z)-t\right]
$$

Since the filling time of the structure is $\sim 314 \mathrm{~ns}$ and the bunch separation is $T_{b}=16 \mathrm{~ns}$, the wake reaches a steady state after the passage of 20 bunches. If we consider a finite set of instants $t_{n}=n T_{b}(n=0,1, \ldots, 19)$, it is possible to take snapshots of the wake every time a bunch arrives. All the functions $W\left(z, n T_{b}\right)$ have been calculated and the snapshots of the single bunch wake at $t=n T_{b}$ have been shown in Fig. 33. The wake experienced by the 20th bunch due to its own interaction with the structure is given, according to the beam loading theorem, by a half of the single bunch potential, i.e. the one shown in Fig. 33.

The total wake ( $\left.W_{\text {TОт }}\right)$ induced by a train of 20 equal bunches, normalized to their charge, is equal to the sum of all the contributions shown in Fig. 33 and it has been reported in Fig. 34.

The field profile in steady state beam loading (whose average is designed to be $E_{a v g}=33 \mathrm{MV} / \mathrm{m}$ ) is defined as the difference of the nominal electric field in the structure and the total wake multiplied by the bunch charge $\left(E_{\text {full_BL }}=E_{\text {nom }}-W_{\text {TOT }} \cdot Q_{\text {bunch }}\right)$.

Assuming $Q_{\text {bunch }}=250 \mathrm{pC}$ the steady state beam loading field profile corresponding to an average accelerating field of $33 \mathrm{MV} / \mathrm{m}$ is shown in Fig. 35. Clearly, the beam loading effects could be fully compensated if the structure is preloaded from the klystron with an electric field profile matching the one shown in Fig. 35.

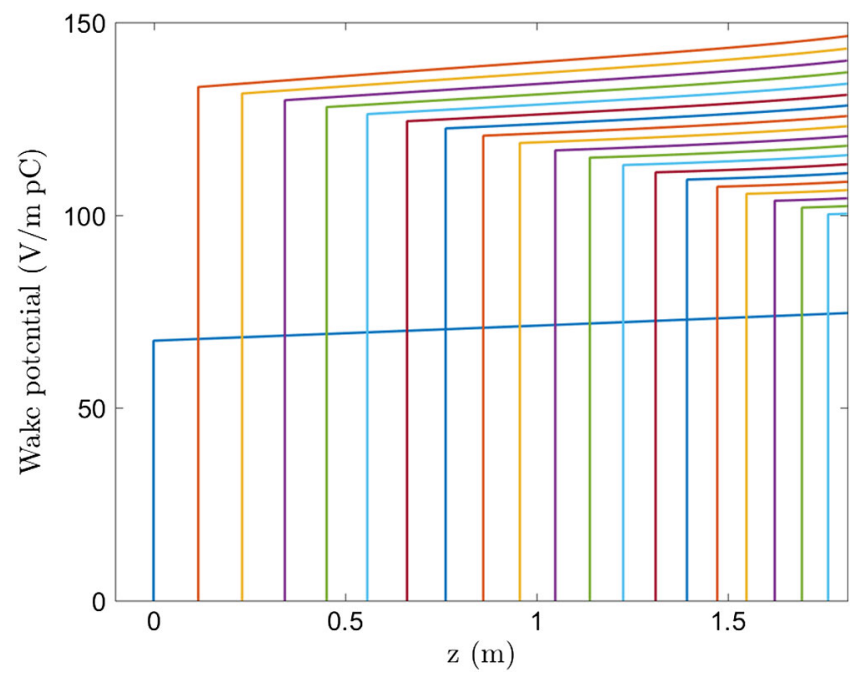

FIG. 33. Wake propagation inside the structure observed at different instants $t_{n}$. 


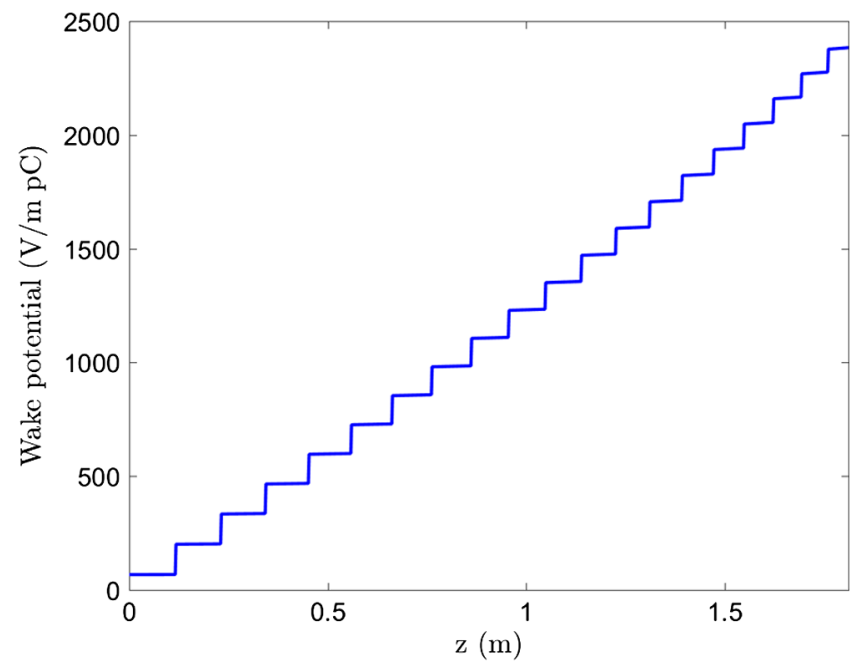

FIG. 34. Total wake potential in steady state beam loading normalized to the bunch charge.

Then, the rf power along the structure is given by:

$$
P_{\text {full_BL }}(z)=E_{\text {full_BL }}(z)^{2} / Z
$$

corresponding to an input power profile as a function of time of:

$$
P_{\text {in }}(-t)=P_{\text {full_BL }}[f(t)] \cdot e^{\frac{\omega_{\mathrm{RF}}}{Q \text { Qavg }} t}
$$

In conclusion, the input power profile reported in Fig. 36 provides the same average accelerating field of $33 \mathrm{MV} / \mathrm{m}$ to each bunch, regardless of its position along the train.

As the bunch train enters in the perfectly preloaded structure, the drive power has to be kept constant for the entire duration of the beam pulse.

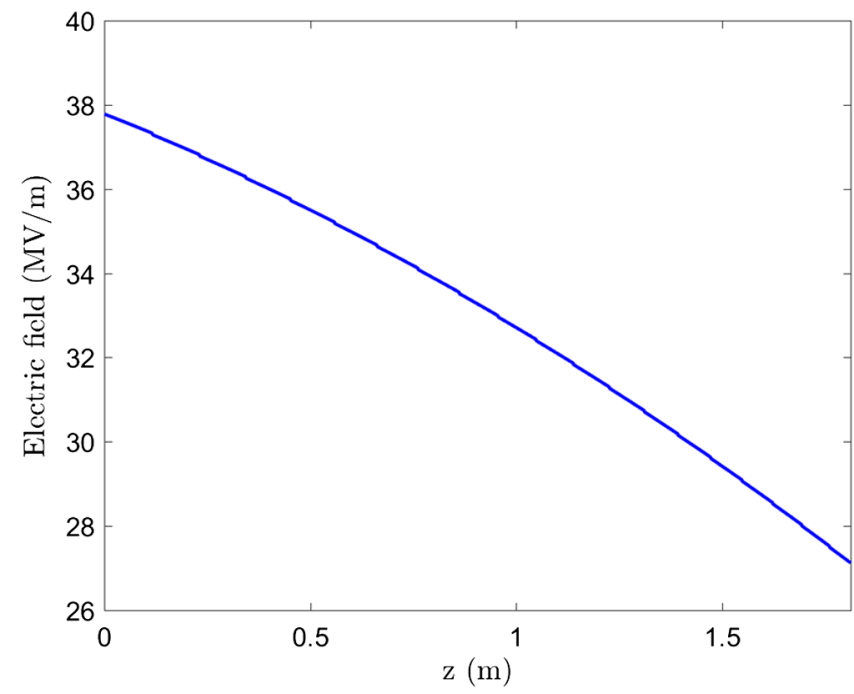

FIG. 35. Accelerating field profile along the structure in steady state beam loading $\left(\mathrm{E}_{\mathrm{avg}}=33 \mathrm{MV} / \mathrm{m}\right)$.

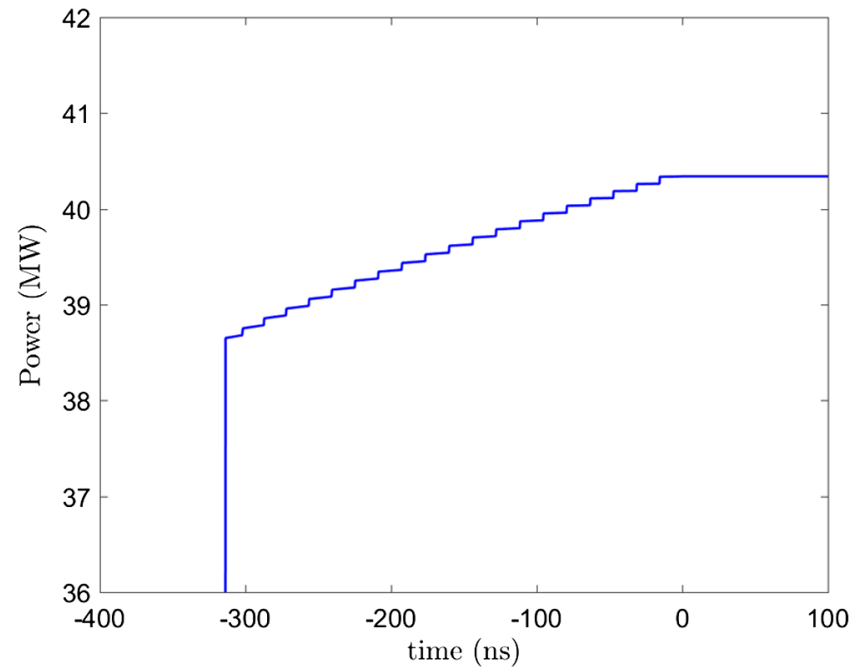

FIG. 36. rf input power profile as a function of time for beam loading full compensation.

\section{APPENDIX C: COUPLER QUADRUPOLE FIELD COMPONENTS ANALYSIS}

As illustrated in Sec. III F, the quadrupole field components in the coupling cells have been minimized looking at the magnitude of the magnetic field in the center of the coupler itself. In the text we pointed out that, first of all, the magnetic field gives the largest contribution to the quadrupole component of the transverse Lorentz force in the coupler itself; secondly, the minimization of this component in the center of the coupler corresponds to a minimization of the quadrupole component along the overall coupler.

The two affirmations can be easily verified simply considering, as example, the geometry of the input coupler given in Fig. 37. The electric and magnetic field components have been calculated on lines 1 and 2 on the two orthogonal planes yz and $\mathrm{xz}$ respectively and at a distance $\mathrm{r}=2 \mathrm{~mm}$ from the axis.

The equivalent quadrupole gradients due to the $\mathrm{E}$ and $\mathrm{B}$ fields [that for the $\mathrm{B}$ field corresponds to the term $\mathrm{A}_{2}$ of Eq. (3)] obtained calculating the absolute values of the transverse forces along the longitudinal coordinates can be approximated by:

$$
\begin{array}{ll}
g_{E} \cong \frac{\left|E_{y \_ \text {line } 1}\right|-\left|E_{x \_ \text {line } 2}\right|}{2 c r} & {\left[\frac{T}{m}\right]} \\
g_{B} \cong \frac{\left|B_{x \_ \text {line1 } 1}\right|-\left|B_{y \_ \text {line } 2}\right|}{2 r} & {\left[\frac{T}{m}\right] .}
\end{array}
$$

$\mathrm{g}>0$ means a focusing quadrupole on the $x$ plane. These two quantities have been plotted, for the input coupler, in Fig. 38 in the two cases with and without the racetrack geometry (for the output coupler we obtain similar results). 


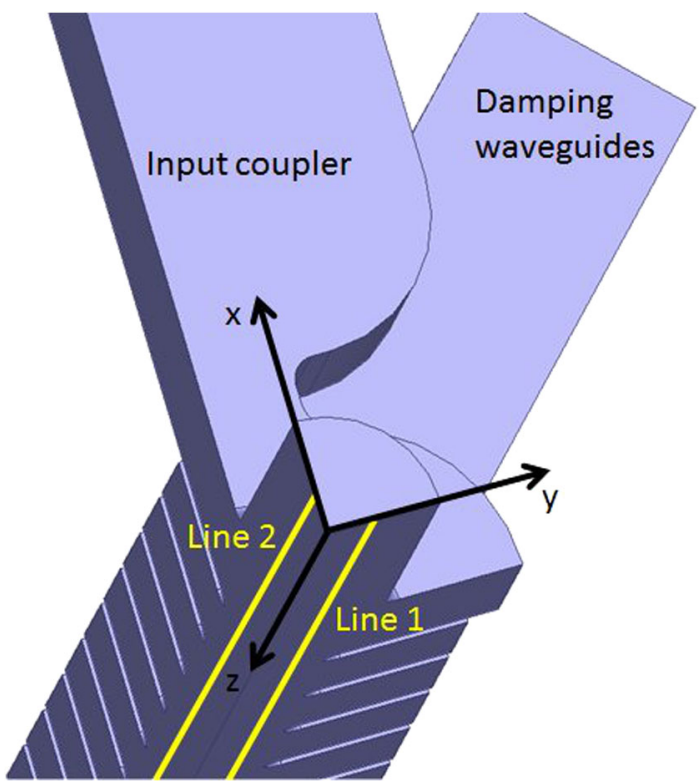

FIG. 37. Input coupler geometry with lines for transverse field calculations.

They clearly show that, first of all, the magnetic contribution dominates, and that the minimization of the gradient in the center of the coupler gives a minimization in the component along the coupler cell.

It is nevertheless important to point out that the transverse forces on the two planes are rf forces and that the total integrated forces along the coupler depend on the particle injection phase $\left(\phi_{\text {inj }}\right)$. The effective equivalent quadrupole gradients due to the electric and magnetic fields that take into account the injection phase and transit time of the particle can be then approximated as:

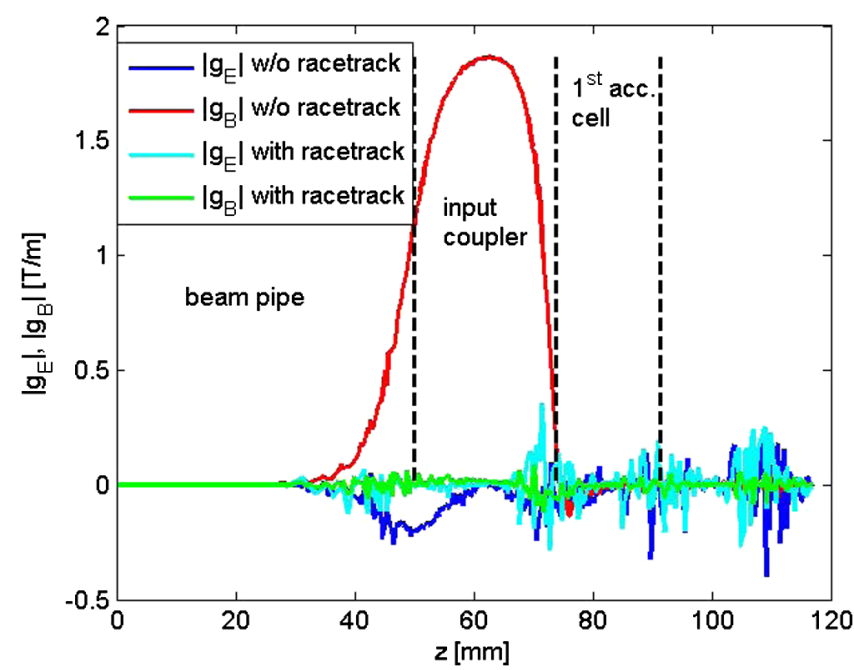

FIG. 38. Equivalent quadrupole gradients due to the $\mathrm{E}$ and $\mathrm{B}$ fields along the coupler. Integrated gradients $\mathrm{G}_{E} \cong-4 \times 10^{-3}[\mathrm{~T}]$, $\mathrm{G}_{B} \cong 4 \times 10^{-2}[\mathrm{~T}]$ (without racetrack), $\mathrm{G}_{E} \cong 0, \mathrm{G}_{B} \cong 0$ (with racetrack).

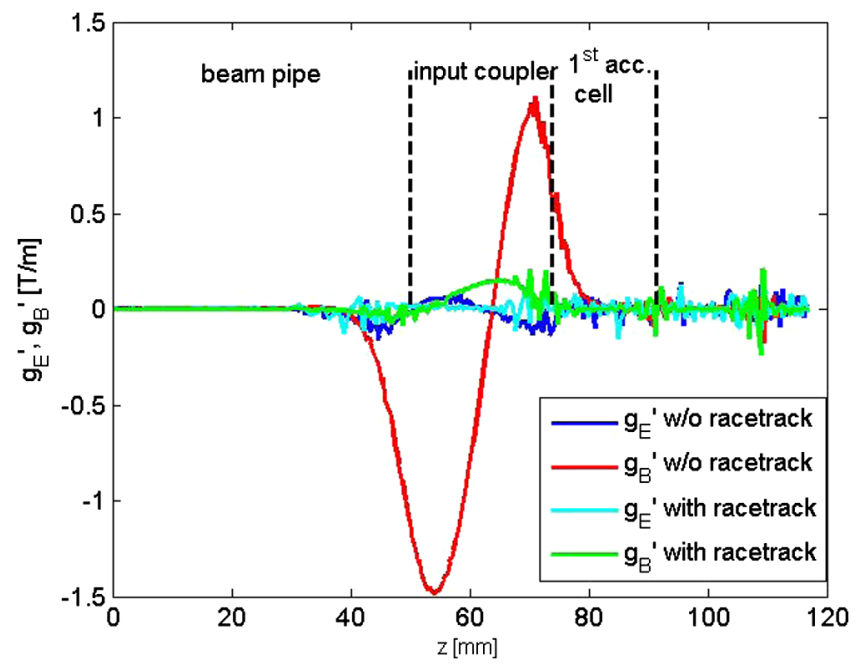

FIG. 39. Equivalent quadrupole gradients due to the E and B fields with transit time factor for an on-crest particle. Integrated gradients $\mathrm{G}_{E}^{\prime} \cong-1 \times 10^{-3}[\mathrm{~T}], \mathrm{G}_{B}^{\prime} \cong-1 \times 10^{-2}[\mathrm{~T}]$ (without racetrack), $\mathrm{G}_{E}^{\prime} \cong 0, \mathrm{G}_{B}^{\prime} \cong 0$ (with racetrack).

$\begin{array}{ll}g_{E}^{\prime} \cong \frac{\operatorname{Re}\left[\left(E_{y \_ \text {line } 1}-E_{x \_ \text {line2 }}\right) e^{j\left(\omega_{\mathrm{RF}} \frac{z}{c}+\varphi_{\text {inj }}\right)}\right]}{2 c r} & {\left[\frac{T}{m}\right]} \\ g_{B}^{\prime} \cong \frac{\operatorname{Re}\left[\left(B_{x \_ \text {line } 1}+B_{y \_ \text {line } 2}\right) e^{j\left(\omega_{\mathrm{RF}_{c}}+\varphi_{\text {inj }}\right)}\right]}{2 r} & {\left[\frac{T}{m}\right]}\end{array}$

As an example these two quantities have been calculated for an injection phase that corresponds to a perfect on crest accelerating field along the structure. The results are given in Fig. 39. The plot shows that, also considering the transit time and the rf field, the racetrack geometry allows to minimize the quadrupole gradients. It is also possible to calculate the integrated gradients according to the formulas:

$$
\begin{aligned}
G_{E} & =\int_{\text {coupler }}\left|g_{E}\right| d z ; & G_{B} & =\int_{\text {coupler }}\left|g_{B}\right| d z \quad[T] \\
G_{E}^{\prime} & =\int_{\text {coupler }} g_{E}^{\prime} d z ; & G_{B}^{\prime} & =\int_{\text {coupler }} g_{B}^{\prime} d z \quad[T]
\end{aligned}
$$

Their values are given in the figure captions.

[1] O. Adriani et al., Technical design report EuroGammaS proposal for the ELI-NP gamma beam system scientific Editor L. Serafini, arXiv:1407.3669.

[2] M. Ferrario et al., Experimental Demonstration of Emittance Compensation with Velocity Bunching, Phys. Rev. Lett. 104, 054801 (2010).

[3] T. Shintake, The choke mode cavity, Jpn. J. Appl. Phys. 31, L1567 (1992).

[4] T. Shintake, HOM-free linear accelerator structure using choke mode cavity, in Proceedings of the 17th Linear Accelerator Meeting, Sendai, Japan, 1992; KEK Report No. 92-66, 1992. 
[5] T. Shintake, Design of high power model of damped linear accelerating structure using choke mode cavity, in Proceedings of the Particle Accelerator Conference, Washington, DC, 1993 (IEEE, New York, 1993).

[6] A. Mosnier, Instabilities in linacs, CAS-CERN Accelerator School: 5th Advanced Accelerator Physics Course, Rhodes, Greece, 1993, pp. 459-514.

[7] K. Yokoya, DESY Report No. 86-084, 1989.

[8] D. Schulte, Multi-bunch calculations in the CLIC main linac, in Proceedings of the 23rd Particle Accelerator Conference, Vancouver, Canada, 2009 (IEEE, Piscataway, NJ, 2009), FR5RFP055.

[9] I. Nesmiyan et al., Beam Dynamics studies for the CLIC main LINAC, IPAC2012/papers/tuppr039.

[10] R. M. Jones et al., Wakefield band partitioning in linac structures, in Proceedings of the 21st International Linac Conference, Gyeongju, Korea, 2002 (Pohang, Accelerator Laboratory, Pohang, Korea, 2002).

[11] R. M. Jones, A study of higher-band dipole wakefields in $\mathrm{X}$-band accelerating structures for the G/NLC, in Proceedings of the 22nd Linear Accelerator Conference (LINAC04), Lubeck, Germany, 2004, Report No. SLACPUB 10682.

[12] A. Bacci et al., Electron Linac design to drive bright Compton back-scattering gamma-ray sources, J. Appl. Phys. 113, 194508 (2013).

[13] D. Alesini et al., The $C$-band accelerating structures for SPARC photoinjector energy upgrade, J. Instrum. 8, P05004 (2013).

[14] R. M. Jones, Wakefield suppression in high gradient linacs for lepton linear colliders, Phys. Rev. ST Accel. Beams 12, 104801 (2009).

[15] A. Grudiev and W. Wuensch, Design of an X-band accelerating structure for the CLIC main linac, in Proceedings of LINAC08, Victoria, BC, Canada, 2008, p. 933.

[16] D. Alesini et al., The damped C-band RF structures for the European ELI-NP proposal, in Proceedings of the 4th International Particle Accelerator Conference, IPAC2013, Shanghai, China, 2013 (JACoW, Shanghai, China, 2013), Vol. 13, WEPFI013.

[17] https://www.ansys.com.

[18] https://www.gdfidl.de.

[19] https://www.cst.com.

[20] G. De Michele, Ph.D. thesis, École Polytechnique Fédérale de Lausanne, 2014.

[21] G. De Michele et al., Broadband electromagnetic characterization of materials for accelerator components, in Proceedings of the 2nd International Particle Accelerator Conference, San Sebastián, Spain (EPS-AG, Spain, 2011), p. 769.

[22] G. De Michele and A. Grudiev, Analysis of long-range wakefield in CLIC main LINAC accelerating structures with damping loads, in Proceedings of the 3rd International Particle Accelerator Conference, New Orleans, LA, 2012 (IEEE, Piscataway, NJ, 2012), p. 3111.

[23] See as example, T. P. Wangler, Principle of rf Linear Accelerators (John Wiley and Sons, Inc., New York, 1998), Chap. 3.

[24] Stanford Two-Miles Accelerator, edited by R. B. Neal (W.A. Benjamin, Inc., New York, 1968).
[25] R. Ganter et al., SwissFEL conceptual design report Report No. 10-04, 2010.

[26] R. Zennaro et al., Conceptual design of the $C$-band module for the SwissFEL, in Proceedings of the 25th International Linear Accelerator Conference, LINAC2010, Tsukuba, Japan (KEK, Tsukuba, Japan, 2010).

[27] D. Farkas et al., SLED: A method of doubling SLAC's energy Report No. SLAC-PUB-1453 1974.

[28] D. Alesini et al., Design, realization and test of C-band accelerating structures for the SPARC_LAB linac energy upgrade, Nucl. Instrum. Methods Phys. Res., Sect. A 837, 161 (2016).

[29] T. Inagaki, C. Kondo, H. Maesaka, T. Ohshima, Y. Otake, T. Sakurai, K. Shirasawa, and T. Shintake, High-gradient $C$-band linac for a compact x-ray free-electron laser facility, Phys. Rev. ST Accel. Beams 17, 080702 (2014).

[30] V. A. Dolgashev, Rf breakdown in normal conducting single-cell structures, in Proceedings of the 21st Particle Accelerator Conference, Knoxville, TN, 2005 (IEEE, Piscataway, NJ, 2005), p. 595.

[31] D. Alesini, Power coupling, CERN Yellow Report No. CERN-2011-007, Geneva, pp. 125-147; arXiv:1112 .3201 .

[32] D. Alesini, A. Gallo, B. Spataro, A. Marinelli, and L. Palumbo, Design of couplers for traveling wave RF structures using 3D electromagnetic codes in the frequency domain, Nucl. Instrum. Methods Phys. Res., Sect. A 580, 1176 (2007).

[33] J. Haimson, B. Mecklenburg, and E. L. Wright, A racetrack geometry to avoid undesirable azimuthal variations of the electric field gradient in high power coupling cavities for TW structures, AIP Conf. Proc. 398, 898 (1997).

[34] P. K. Ambattu, G. Burt, A. Grudiev, V. Dolgashev, and A. Dexter, Coupler induced monopole component and its minimization in deflecting cavities, Phys. Rev. ST Accel. Beams 16, 062001 (2013).

[35] D. Alesini, A. Citterio, G. Campogiani, L. Ficcadenti, M. Migliorati, A. Mostacci, L. Palumbo, S. Persichelli, and R. Zennaro, Tuning procedure for traveling wave structures and its application to the C-Band cavities for SPARC photo injector energy upgrade, J. Instrum. 8, P10010 (2013).

[36] V. Pettinacci et al., Thermal-Mechanical Analysis of the RF Structures for the ELI-NP proposal, Proceedings of IPAC 2014, JACoW, Geneva, Switzerland (2014), p. 3860.

[37] S. Bini et al., SPARC-RF-14/001 technical note, 2014, available at http://www.lnf.infn.it/acceleratori/sparc/ TECHNOTES/RF/SPARC_RF_14_001.pdf.

[38] D. Boussard, Beam loading (particle accelerators), CASCERN, Accelerator School: 5th Advanced Accelerator Physics Course, Rhodes, Greece, 1993 (1995), pp. 415-436.

[39] J. W. Wang, SLAC Report No. 339, 1989.

[40] S. Liu, M. Fukuda, S. Araki, N. Terunuma, J. Urakawa, K. Hirano, and N. Sasao, Nucl. Instrum. Methods Phys. Res., Sect. A 584, 1 (2008).

[41] C. Adolphsen et al., Beam loading compensation in the NLCTA, in Proceedings of the Particle Accelerator Conference, Vancouver, BC, Canada, 1997 (IEEE, New York, 1997).

[42] A. Lunin, V. Yakovlev, and A. Grudiev, Analytical solutions for transient and steady state beam loading in 
arbitrary traveling wave accelerating structures, Phys. Rev. ST Accel. Beams 14, 052001 (2011).

[43] O. Kononenko and A. Grudiev, Transient beam-loading model and compensation in Compact Linear Collider main linac, Phys. Rev. ST Accel. Beams 14, 111001 (2011).

[44] I. Syratchev and T. Higo, KEK Report No. 96-8, 1996.

[45] M. Satoh, T. Matsumoto, T. Shidara, S. Fukuda, H. Kobayashi, Y. Kamiya, N. Nakamura, T. Koseki, and S. Miura, Initial-beam-loading compensation system for high-intensity electron linacs, Nucl. Instrum. Methods Phys. Res., Sect. A 538, 116 (2005).

[46] M. Satoh, T. Koseki, T. Shidara, S. Fukuda, H. Kobayashi, Y. Kamiya, N. Nakamura, T. Sakai, Y. Hayakawa, T. Tanaka, K. Hayakawa, I. Sato, and S. Miura, Development of a new initial-beam-loading compensation system and its application to a free-electron-laser linac, Phys. Rev. ST Accel. Beams 12, 013501 (2009).

[47] R. M. Jones, V. A. Dolgashev, and J. W. Wang, Dispersion and energy compensation in high-gradient linacs for lepton colliders, Phys. Rev. ST Accel. Beams 12, 051001 (2009).

[48] D. Alesini, S. Bini, R. Di Raddo, V. Lollo, L. Pellegrino, L. Ficcadenti, V. Pettinacci, L. Palumbo, and L. Serafini, Design and RF Test of Damped C-Band Accelerating Structures for the ELI-NP LINAC, in Proceedings of IPAC2014, Dresden, Germany, 2014 (JACoW, CERN, Geneva, 2014), p. 3856.

[49] http://www.comeb.it/.

[50] http://www.morganbrazealloys.com/. 